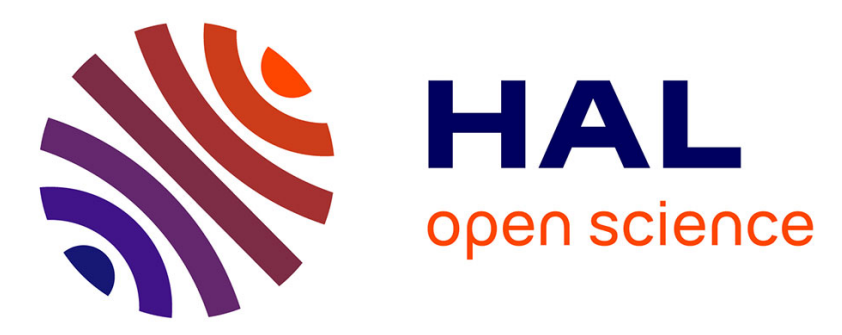

\title{
Stochastic continuum modeling of random interphases from atomistic simulations. Application to a polymer nanocomposite
}

Thinh-Tien Le, Johann Guilleminot, Christian Soize

\section{- To cite this version:}

Thinh-Tien Le, Johann Guilleminot, Christian Soize. Stochastic continuum modeling of random interphases from atomistic simulations. Application to a polymer nanocomposite. Computer Methods in Applied Mechanics and Engineering, 2016, 303, pp.430-449. 10.1016/j.cma.2015.10.006 . hal01223801

\section{HAL Id: hal-01223801 \\ https://hal.science/hal-01223801}

Submitted on 3 Nov 2015

HAL is a multi-disciplinary open access archive for the deposit and dissemination of scientific research documents, whether they are published or not. The documents may come from teaching and research institutions in France or abroad, or from public or private research centers.
L'archive ouverte pluridisciplinaire HAL, est destinée au dépôt et à la diffusion de documents scientifiques de niveau recherche, publiés ou non, émanant des établissements d'enseignement et de recherche français ou étrangers, des laboratoires publics ou privés. 


\title{
Stochastic continuum modeling of random interphases from atomistic simulations. Application to a polymer nanocomposite
}

\author{
T.T. Le ${ }^{\mathrm{a}}$, J. Guilleminot ${ }^{\mathrm{a}, *}$, C. Soize $\mathrm{a}^{\mathrm{a}}$ \\ ${ }^{a}$ Université Paris-Est, Laboratoire Modélisation et Simulation Multi Echelle, MSME UMR 8208 CNRS, 5 bd \\ Descartes, 77454 Marne-la-Vallée, France
}

\begin{abstract}
This paper is concerned with the probabilistic multiscale analysis of polymeric materials reinforced by nanoscopic fillers. More precisely, this work is devoted to the stochastic modeling and inverse identification of the random field associated with the elastic properties in the so-called interphase region. For illustration purposes, a prototypical polymer system reinforced by a Silica nanoscopic inclusion is considered. Molecular Dynamics (MD) simulations are first performed and used to characterize the conformational properties of the polymer chains in the neighborhood of the inclusion. It is shown that these chains are characterized by a specific tangential orientation which, together with the density profile and variations in chain mobility, allows for the geometric definition of the interphase region. Mechanical virtual testing is next completed on a set of initial configurations, hence providing a simulated database for model calibration. The results thus obtained are subsequently used to construct a random field model for the interphase stiffness. An inverse calibration procedure is finally proposed and relies on a stated equivalence between the apparent properties obtained from MD simulations and those computed by numerical homogenization in the continuum mechanics formulation. The interphase elasticity random field is seen to exhibit nonnegligible fluctuations, and the estimates of parameters related to spatial correlation are shown to be consistent with characteristic lengths of the atomistic model, such as the interphase thickness.
\end{abstract}

Keywords: Interphase, maximum entropy, Molecular Dynamics, nanocomposite, probabilistic model, random field, inverse problem

\footnotetext{
"Preprint - Accepted for publication in Computer Methods in Applied Mechanics and Engineering, available online on October 20, 2015 (doi:10.1016/j.cma.2015.10.006).

${ }^{*}$ Corresponding author

Email address: johann.guilleminot@u-pem.fr (J. Guilleminot)
} 


\section{Introduction}

The prediction of the enhanced physical properties exhibited by heterogeneous materials reinforced with nanoscopic fillers is a great challenge of nanoscale science. For such systems, small-scale interactions (between the fillers and the matrix phase, or between adjacent fillers) are no longer negligible and must be accounted for in a multiscale setting. One difficulty pertaining to modeling issues is that the final properties strongly depend on the coupling between various parameters, such as size effects or filler aggregation $[1,2]$. Perhaps one the most discussed specificity of nanoreinforced composites is the existence of an interphase region surrounding the inclusions. Such a region is commonly identified with the region confined to inclusion neighborhood where the polymer chains exhibit conformational and geometrical properties that are substantially different from those in the bulk polymer phase. The experimental characterization of such properties, by using high-resolution nuclear magnetic resonance (NMR) techniques for instance, gave rise to an extensive literature: see e.g. [3, 4, 5, 6, 7, 8, 9] for systems reinforced with Silica nanoinclusions, and [10] for similar results with carbon black fillers. All these results show a local disturbance in the mobility of the polymer chains, as well as density fluctuations vanishing away from the inclusions. These observations are well correlated by atomistic simulations, which have additionaly highlighted a preferred orientation of the polymer chain segments tangentially to the particle surface; see $[11,12,13,14,15,16,17,18,19]$ for Molecular Dynamics (MD) simulations, as well as $[20,21,22,23,24]$ for the use of a Monte Carlo solver.

From a mechanical point of view, two types of approach were developed so as to estimate the effective properties of nanocomposites while accounting for size effects. A first class of methods relies on the integration of so-called interface models, such as spring-layer or membrane-type imperfect interface ones, in various extensions of classical (e.g. Eshelby-type) micromechanical frameworks. In this case, surface and interfacial energy concepts are invoked and plugged into asymptotic formulations, hence allowing for an explicit dependance of effective moduli on the nanoscale characteristic length (the radius of a spherical filler, for instance); see [25, 26, 27, 28] and the references therein to name a few. A major issue related to such approaches is the definition of the interface stiffness, which may not be necessarily positive-definite (see [29] for a derivation based on MD simulations, for instance). In the second class of models, the interphase is considered as an additional phase with a finite volume and standard (in the thermodynamical sense) mechanical properties. Such formulations can be subsequently used within (semi-)analytical homogenization schemes, such as the generalized self consistent scheme [15, 12,30,31], or coupled with computational approaches [32]. It should be noted that these approaches are sequential, in the sense that they rely on an a priori characterization of interphase properties. Energy-based coupling techniques were alternatively developed in order to couple, in a concurrent manner, an atomistic description of the interphase with a finite element formulation (see [33] and the references therein, for instance).

Despite the intrinsic stochastic nature of the physical phenomena occurring while processing 
nanocomposites, most of the multiscale models developed so far are deterministic and presume that the interphase properties are known. More recently, a few studies involving some uncertainty quantification aspects were proposed. In a series of papers, $\mathrm{Vu}$ Bac and coworkers have investigated sensitivity analysis of multiscale predictions by using forward propagation of parametric uncertainties related either to input parameters of MD simulations or to other parameters (such as the volume fraction of fillers) in a continuum mechanics formulation [34, 35, 36, 37] - see also $[38,39]$ for the modeling of MD finite sampling noise and uncertainties in force field parameters, as well as [40] for a study where uncertainties due to MD finite sampling are exchanged back-and-forth between an atomistic model and a continuum one. In [41], an inverse identification procedure for some parameters modeled as random variables (such as particle radius or distribution, or matrix mechanical properties) is proposed. Finally, uncertainty propagation from nanoscale to structural analysis has been performed in [42].

This work is devoted to the complementary and fundamental aspect of model construction and inverse calibration based on atomistic simulations. Here, and contrary to previous works developed elsewhere, the interphase elastic properties are modeled by a non-Gaussian tensor-valued random field. MD simulations are used so as to infer some basic properties of the latter, such the material symmetry locally exhibited or the underlying correlation structure, and to construct a virtual database devoted to the model calibration. This identification step is subsequently addressed by solving a statistical inverse problem stating the equivalence of the apparent properties obtained from atomistic computations and those estimated from stochastic homogenization in a continuum mechanics formulation.

This paper is organized as follows. Section 2 is devoted to the definition and virtual mechanical testing of the nanosystem under consideration. In particular, the atomistic models are introduced, together with the assembly procedure for the nanocomposite. The stochastic model is then introduced in Section 3. The methodology for inverse calibration is finally presented in Section 4.

\section{Modeling and virtual testing of model nanocomposites}

This section is devoted to the modeling and mechanical testing of a prototypical nanocomposite made up of a polymer matrix filled with a silica nanoinclusion. The overall methodology associated with the MD simulations and related issues is mostly borrowed from [12]. The atomistic descriptions of the polymer and silica particle are first introduced in Sec. 2.1 and Sec. 2.2, respectively. The assembly procedure of the nanostructure is then summarized in Sec. 2.3. The framework for virtual testing and results are finally presented in Sec. 2.4. All MD simulations are carried out in the LAMMPS package [43], with a time step set to 2 fs. Atomistic images are produced by using the OVITO program [44].

Let $\boldsymbol{r}^{i}$ denote the position of atom $i, 1 \leqslant i \leqslant N$, and let $\boldsymbol{r}=\left(\boldsymbol{r}^{1}, \ldots, \boldsymbol{r}^{N}\right)$. Let $\boldsymbol{r}^{i j}=\boldsymbol{r}^{j}-\boldsymbol{r}^{i}$ and $r^{i j}=\left\|\boldsymbol{r}^{i j}\right\|$. The notation $i-j$ is used hereafter to denote the bond between atoms $i$ and $j$. 


\subsection{Molecular model of polymer matrix}

In this study, a prototypical generic model of an amorphous linear polymer is considered. More specifically, the system under consideration is made up of $n_{\text {pc }}$ polymer chains, each of which containing 1,000 united-atom $\mathrm{CH}_{2}$ sites.

\subsubsection{Force field description}

The functional form of the force field in the polymer system classically reads as

$$
\mathcal{V}(\boldsymbol{r})=\mathcal{V}_{\mathrm{B}}(\boldsymbol{r})+\mathcal{V}_{\mathrm{UB}}(\boldsymbol{r}),
$$

where

$$
\mathcal{V}_{\mathrm{B}}(\boldsymbol{r})=\sum_{\{i, j\} \in \mathbb{B}_{b}} \mathcal{V}_{b}\left(r^{i j}\right)+\sum_{\{i, j, k\} \in \mathbb{B}_{\theta}} \mathcal{V}_{\theta}\left(\theta^{i j k}\right)+\sum_{\{i, j, k, \ell\} \in \mathbb{B}_{\phi}} \mathcal{V}_{\phi}\left(\phi^{i j k \ell}\right)
$$

is a term modeling interactions between $m$-uplets of bonded atoms $(2 \leqslant m \leqslant 4)$, and

$$
\mathcal{V}_{\mathrm{UB}}(\boldsymbol{r})=\sum_{i=1}^{N} \sum_{j>i} \mathcal{V}_{\mathrm{LJ}}\left(r^{i j}\right)
$$

models non-bonded interactions. In Eq. (2), $\theta^{i j k}$ denotes the angle $\left(\widehat{\boldsymbol{r}^{j i}, \boldsymbol{r}^{j k}}\right)$ between bonds $i-j$ and $j-k$, and $\phi^{i j k \ell}$ is the angle between the planes spanned by vector pairs $\left(\boldsymbol{r}^{i j}, \boldsymbol{r}^{k j}\right)$ and $\left(\boldsymbol{r}^{j k}, \boldsymbol{r}^{\ell k}\right)$. The sets $\mathbb{B}_{b}, \mathbb{B}_{\theta}$ and $\mathbb{B}_{\phi}$ are the sets of pairs, triplets and quadruplets of bonded atoms along a polymer chain, all chains being considered. Therefore, $\operatorname{card}\left(\mathbb{B}_{b}\right)=n_{\mathrm{pc}}\left(n_{\mathcal{P}}-1\right), \operatorname{card}\left(\mathbb{B}_{\theta}\right)=n_{\mathrm{pc}}\left(n_{\mathcal{P}}-2\right)$ and $\operatorname{card}\left(\mathbb{B}_{\phi}\right)=n_{\mathrm{pc}}\left(n_{\mathcal{P}}-3\right)$, with $n_{\mathcal{P}}$ the degree of polymerization. The harmonic potentials $\mathcal{V}_{b}$ and $\mathcal{V}_{\theta}$ are defined as follows:

$$
\begin{gathered}
\mathcal{V}_{b}(r)=\frac{1}{2} K_{b}\left(r-r_{0}\right)^{2}, \\
\mathcal{V}_{\theta}(\theta)=\frac{1}{2} K_{\theta}\left(\cos (\theta)-\cos \left(\theta_{0}\right)\right)^{2},
\end{gathered}
$$

where $r_{0}$ and $\theta_{0}$ are the equilibrium bond length and bond angle, and $K_{b}$ and $K_{\theta}$ are model parameters. The torsional potential is given by

$$
\mathcal{V}_{\phi}(\phi)=\sum_{i=1}^{5} A_{m} \cos ^{m-1}(\phi),
$$

with $\left\{A_{m}\right\}_{i=1}^{5}$ a set of additional model parameters. Nonbonded pair interactions are governed by a truncated Lennard-Jones 12-6 potential:

$$
\begin{aligned}
\mathcal{V}_{\mathrm{LJ}}(r) & =4 \varepsilon\left(\left(\frac{\sigma}{r}\right)^{12}-\left(\frac{\sigma}{r}\right)^{6}\right), \quad r \leqslant r_{c} \\
& =0, \quad r>r_{c}
\end{aligned}
$$


where the well depth $\varepsilon$ and collision diameter $\sigma$ are parameters depending on the polymer system, and $r_{c}$ denotes the cutoff radius (here, $r_{c}=1.4 \mathrm{~nm}$ ). The values of the force field parameters for the polymer matrix are listed in Tab. 1 (see $[45,12]$ ).

\begin{tabular}{cccc}
\hline Potential & Parameter & Value & Unit \\
\hline $\mathcal{V}_{b}$ & $K_{b}$ & 700 & $\mathrm{kcal} / \mathrm{mol} / \AA^{2}$ \\
& $b_{0}$ & 0.15 & $\mathrm{~nm}$ \\
\hline $\mathcal{V}_{\theta}$ & $K_{\theta}$ & 124.2830 & $\mathrm{kcal} / \mathrm{mol}$ \\
& $\theta_{0}$ & 112.813 & $\mathrm{deg}$ \\
\hline $\mathcal{V}_{\phi}$ & $A_{1}$ & 2.1109 & $\mathrm{kcal} / \mathrm{mol}$ \\
& $A_{2}$ & 4.3229 & $\mathrm{kcal} / \mathrm{mol}$ \\
& $A_{3}$ & 1.1665 & $\mathrm{kcal} / \mathrm{mol}$ \\
& $A_{4}$ & -7.6004 & $\mathrm{kcal} / \mathrm{mol}$ \\
& $A_{5}$ & 0 & $\mathrm{kcal} / \mathrm{mol}$ \\
\hline $\mathcal{V}_{\mathrm{LJ}}$ & $\sigma$ & 0.43 & $\mathrm{~nm}$ \\
& $\varepsilon$ & 0.1133 & $\mathrm{kcal} / \mathrm{mol}$ \\
\hline
\end{tabular}

Table 1: Force field parameters used for the atomistic modeling of the polymer phase.

\subsubsection{Initial configuration and relaxation steps}

A set of 20 initial configurations is generated for three different systems, corresponding to $n_{\mathrm{pc}} \in\{10,80,320\}$, by using the Self Avoiding Random Walk (SARW) algorithm [46]. In order to obtain fairly good initial configurational properties, MD relaxation steps are alternately performed in the NVT and NPT ensembles, making use of Nosé-Hoover schemes. More precisely, and following [12], simulations are started at $500 \mathrm{~K}$, hence ensuring an amorphous structure through the induced high chain mobility. The latter is illustrated in Fig. 1, for $n_{\mathrm{pc}}=10$. The systems are subsequently cooled down at $100 \mathrm{~K}$, with a cooling rate of $1 \mathrm{~K} / \mathrm{ps}$. A NPT treatment is finally applied at $100 \mathrm{~K}$ and at a target isotropic pressure of 0 bar. It should be noticed that the simulations are performed below the glass transition temperature (which is estimated to $264 \mathrm{~K}$ for $n_{\mathrm{pc}}=10$, following the methodology defined in [12] with a cooling rate of $0.1 \mathrm{~K} / \mathrm{ps}$ ), and that the above procedure yields samples exhibiting conformational characteristics in accordance with the literature.

\subsection{Molecular model of Silica}

In order to create amorphous $\mathrm{SiO}_{2}$ nanoinclusions exhibiting an isotropic mechanical behavior, a set a $\alpha$-quartz silica crystalline structures is first generated. These configurations are then relaxed by using MD simulations with Van der Waals and Coulomb (non-bonded) interactions. The first 

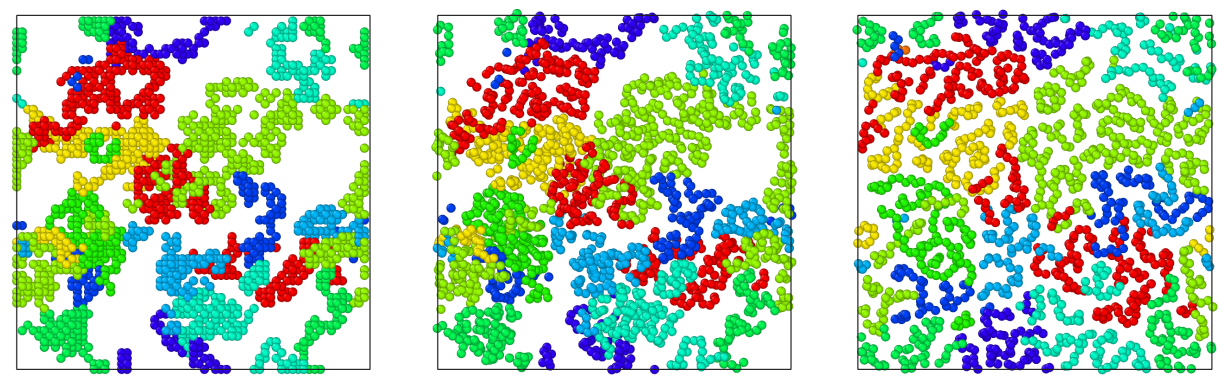

Figure 1: Snapshot of the polymer chains $\left(n_{\mathrm{pc}}=10\right)$ during the MD relaxation step, starting from an initial configuration obtained by the SARW algorithm (from left to right).

type of interactions is modeled by a Buckingham potential:

$$
\mathcal{V}_{B C K}(r)=B_{1} \exp \left\{-\frac{r}{B_{2}}\right\}-\frac{B_{3}}{r^{6}}
$$

where the values of parameters $\left\{B_{i}\right\}_{i=1}^{3}$ are outlined below in Tab. 2 for the three types of bonds encountered in the system (note that the Van der Waals interaction between Si atoms is not taken into account). The Coulomb interaction is modeled by the following potential:

\begin{tabular}{cccc}
\hline Bond & $B_{1}(\mathrm{eV})$ & $B_{2}(\AA)$ & $B_{3}\left(\mathrm{eV} / \AA^{6}\right)$ \\
\hline $\mathrm{Si}-\mathrm{Si}$ & 0 & 0.0657 & 0 \\
\hline $\mathrm{Si}-\mathrm{O}$ & $18,003.7572$ & 0.205204 & 133.5381 \\
\hline $\mathrm{O}-\mathrm{O}$ & $1,388.773$ & 0.36219 & 175 \\
\hline
\end{tabular}

Table 2: Force field parameters used for the atomistic modeling of silica inclusion.

$$
\mathcal{V}\left(r^{i j}\right)=\frac{q_{i} q_{j}}{4 \pi \epsilon_{0} r^{i j}}
$$

where $q_{i}$ and $q_{j}$ are the electric charges of atoms $i$ and $j\left(q_{\mathrm{Si}}=2.4 e\right.$ and $q_{\mathrm{O}}=-1.2 e$, with $e$ the charge of a proton), and $\epsilon_{0}$ is the vacuum permittivity. Amorphous structures are then obtained through a heat up and cool down treatment, down to the equilibrium temperature of $100 \mathrm{~K}$. In order to speed up computations, the above non-bonded interactions are subsequently replaced, at equilibrium, by fictitious bonded ones (see [12] for a discussion). More precisely, bond stretching and angle bending are modeled by using the harmonic potentiels defined in Eqs (4-5), and the values of associated parameters are listed in Tab 3. A comparison of the probability density functions of 


\begin{tabular}{ccccc}
\hline Potential & Bond & Parameter & Value & Unit \\
\hline $\mathcal{V}_{b}$ & $\mathrm{Si}-\mathrm{O}$ & $K_{b}$ & 575.7301 & $\mathrm{kcal} / \mathrm{mol} / A^{2}$ \\
& & $b_{0}$ & 0.1625 & $\mathrm{~nm}$ \\
\hline $\mathcal{V}_{\theta}$ & $\mathrm{Si}-\mathrm{O}-\mathrm{Si}$ & $K_{\theta}$ & 47.8011 & $\mathrm{kcal} / \mathrm{mol}$ \\
& & $\theta_{0}$ & 145 & $\mathrm{deg}$ \\
& $\mathrm{O}-\mathrm{Si}-\mathrm{O}$ & $K_{\theta}$ & 71.7017 & $\mathrm{kcal} / \mathrm{mol}$ \\
& & $\theta_{0}$ & 109.5 & $\mathrm{deg}$ \\
\hline
\end{tabular}

Table 3: Force field parameters used for the atomistic modeling of the silica particle.
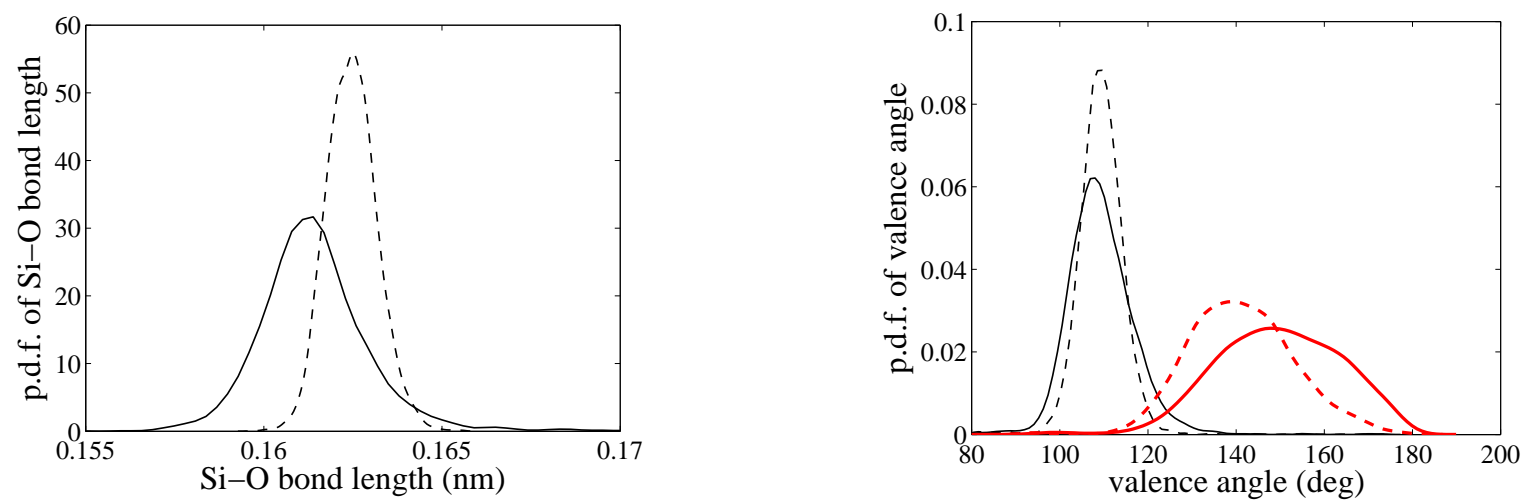

Figure 2: Probability density functions of $\mathrm{Si}-\mathrm{O}$ bond length (left) and $\mathrm{O}-\mathrm{Si}-\mathrm{O}$ (black)/Si-O-Si (red) valence angles (right). Solid line: atomistic model with non-bonded interactions. Dashed line: surrogate atomistic model with bonded interactions.

the $\mathrm{Si}-\mathrm{O}$ bond length and $\mathrm{O}-\mathrm{Si}-\mathrm{O} / \mathrm{Si}-\mathrm{O}-\mathrm{Si}$ valence angles (at equilibrium) obtained from the atomistic simulations with either non-bonded or bonded interactions is shown in Fig. 2. It is seen that the two probability density functions are reasonably close to each other (note that the same conclusion can be drawn for the valence angles). The difference in variance is more significant for the $\mathrm{Si}-\mathrm{O}$ bond length, for which the mean values for the non-bonded and bonded cases turn out to be pretty similar (with values equal to $1.6152 \AA$ and $1.6247 \AA$, respectively). For each value of $n_{\mathrm{pc}}$, spherical nanoparticules of different radii are extracted from MD boxes following the procedure detailed in [12], so that the volume fraction of silica remains constant and equal to $4.8 \%$ regardless of the polymer system. Let $R_{p}$ denote the approximate particle radius. For $n_{\mathrm{pc}}=10$ (resp. $n_{\mathrm{pc}}=80$ and $\left.n_{\mathrm{pc}}=320\right)$, it is found that $R_{p} \approx 1.5 \mathrm{~nm}\left(\operatorname{resp} . R_{p} \approx 3 \mathrm{~nm}\right.$ and $\left.R_{p} \approx 4.8 \mathrm{~nm}\right)$. It is worth noticing that because of the atomistic model, each inclusion is not perfectly spherical and exhibits some roughness, especially for low target diameters. This characteristic, which is illustrated in Fig. 3 (where atomistic models and associated continuum approximations are represented for two different diameters), is an additional source of randomness within the atomistic description. 

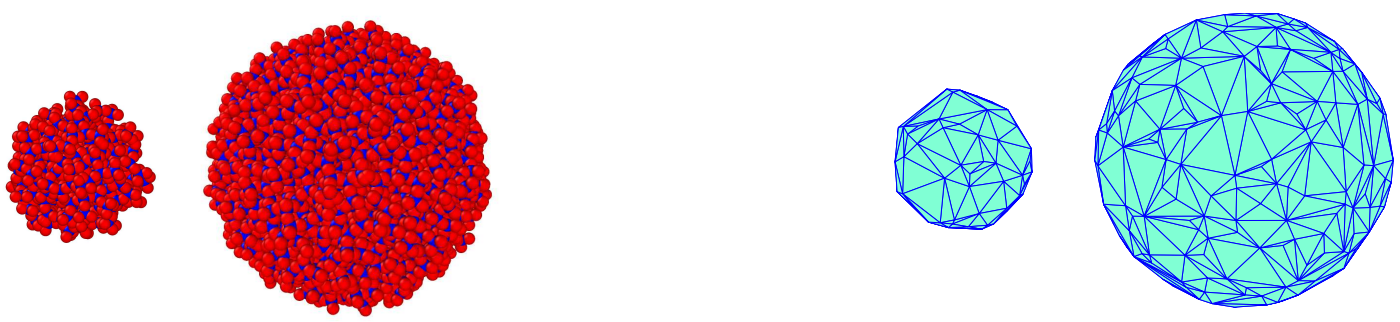

Figure 3: Visualization of nanoparticles: atomistic representations (left) and associated polyhedral representations (right). The smallest (resp. largest) particle corresponds to a diameter equal to $3 \mathrm{~nm}$ (resp. $6 \mathrm{~nm}$ ).

\subsection{Molecular model of nanocomposite}

Once the initial, equilibrated configurations of polymer and silica are obtained, nanocomposite samples are created as follows [13]. First, and for a given configuration of each subsystem, the MD box containing the polymer is swelled by a volume corresponding the one occupied by the particle. Second, a soft repulsive potential is gradually introduced, under NVT conditions, at the center of the box. Finally, the silica particle is inserted once the target hole diameter is reached, hence creating an initial configuration for the nanocomposite. This generation step is then followed by a MD relaxation procedure (similar to the one carried out on the polymer system, with a temperature increase up to $500 \mathrm{~K}$, followed by a final decrease down to $100 \mathrm{~K}$ at a cooling rate of $1 \mathrm{~K} / \mathrm{ps}$ ) where only non-bonded Lennard-Jones interactions between the $\mathrm{CH}_{2}$ sites and oxygen atoms are considered. The force field parameters for $\mathrm{CH}_{2}-\mathrm{O}$ interactions are estimated following the Lorentz-Berthelot mixing rules [47]: $\sigma=0.31 \mathrm{~nm}, \varepsilon=0.1288 \mathrm{kcal} / \mathrm{mol}$. A final relaxation step under NPT conditions is performed during 2 ns at $100 \mathrm{~K}$ and 0 bar. A snapshot of one nanocomposite sample is shown in Fig. 4.

\subsection{Morphological and mechanical characterizations}

\subsubsection{Radial distribution of density}

Here, we investigate the radial evolution of polymer density in the nanocomposite system. To this aim, instantaneous estimations of the local density are obtained on spherical layers of constant volume $V_{\rho}$ and averaged over a sufficiently large time interval $[0, \Delta]$. Each layer is specifically defined by an interior radius $R_{\rho}^{i}$ - the exterior radius $R_{\rho}^{e}$ being determined from the latter and $V_{\rho}$. Let $R_{\rho}^{m}=\left(R_{\rho}^{i}+R_{\rho}^{e}\right) / 2$ be the mean radius of a given layer. The instantaneous local density in the 


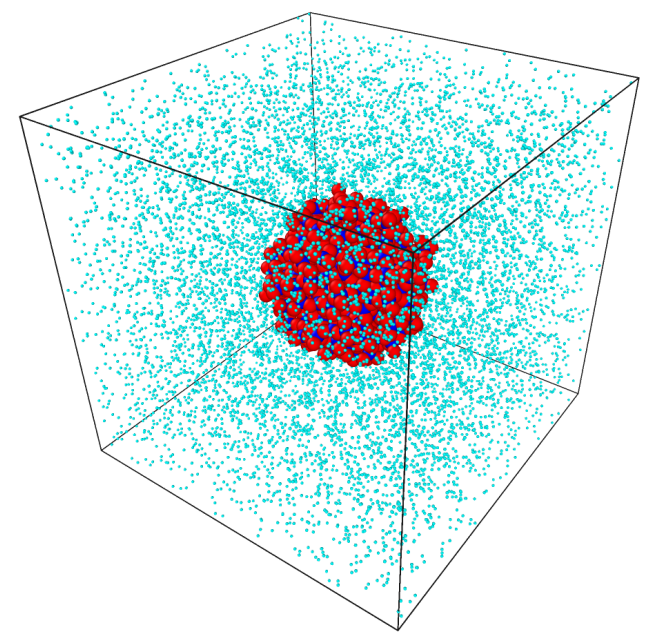

Figure 4: Snapshot of a nanocomposite sample at equilibrium $\left(n_{\mathrm{pc}}=10\right)$. The $\mathrm{CH}_{2}$ sites appear as small particles; the $\mathrm{Si}$ and $\mathrm{O}$ atoms are the blue and red big particles, respectively.

layer defined by mean radius $R_{\rho}^{m}$ is defined as

$$
\widetilde{\rho_{n}}\left(R_{\rho}^{m}, t\right)=\frac{M_{p} N\left(R_{\rho}^{m}, t\right)}{N_{A} V_{\rho}},
$$

where $M_{p}$ is the molar mass of the polymer, $N\left(R_{\rho}^{m}, t\right)$ is the number of $\mathrm{CH}_{2}$ sites in the layer under consideration at time $t$ and $N_{A}$ is the Avogadro constant. The radial density is then given by

$$
\rho_{n}\left(R_{\rho}^{m}\right)=\frac{1}{\Delta} \int_{0}^{\Delta} \widetilde{\rho_{n}}\left(R_{\rho}^{m}, t\right) d t .
$$

Note that in practice, convergence analysis must be performed with respect to both $V_{\rho}$ and $\Delta$. The graphs of $\left(R_{\rho}^{m}-R_{p}\right) \mapsto \rho_{n}\left(R_{\rho}^{m}\right) / \rho_{p}$ is shown in Fig. 5 for different system sizes, where $\rho_{p}$ is the density of pure polymer estimated from MD simulations. Decreasing density oscillations are clearly observed in the neighborhood of the particle, no matter the value of $n_{\mathrm{pc}}$. These fluctuations occur for a distance to the particle surface less than $2 \mathrm{~nm}$, and the density of pure polymer is recovered beyond this limit. It is worth pointing out that the main oscillations are still exhibited at higher temperatures, and that the noise in density estimation increases far from the silica particle. In this region, a tangential reorientation of the polymer segments and a decrease in chain mobility are further observed, hence making the polymer structure much less amorphous. The zone of matrix disturbance thus defined, with a constant thickness equal to $2 \mathrm{~nm}$, is identified with the interphase 


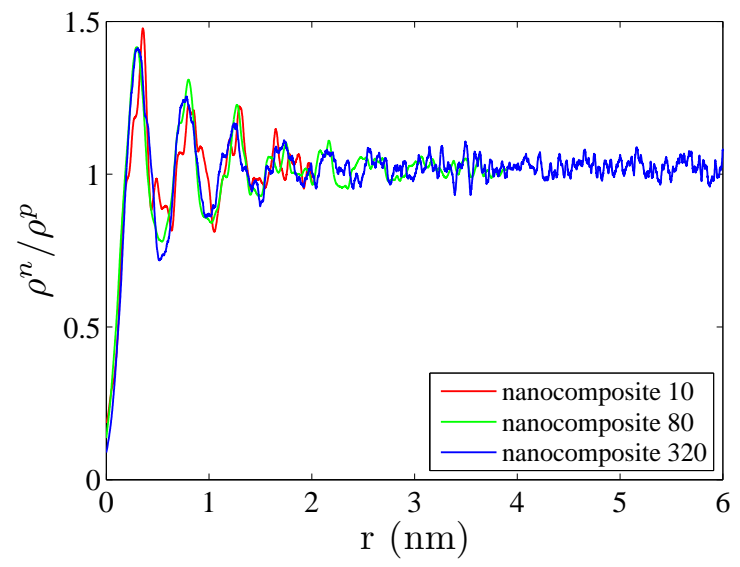

Figure 5: Radial density distribution of mass for three nanocomposite system, obtained from the nanoparticle surface and normalized by mass density of pure polymer $\rho^{p}$ corresponding.

region hereafter. Note that such a definition for the interphase region is not unique and basically depends on the monitored quantities of interest (see [48] for numerical investigations - on a more detailed atomistic model and for another nanocomposite system - and insightful comments on this issue). Whereas this dependence may impact some quantitative results (such as the estimation of the correlation lengths) obtained in the remainder of this paper, it does affect neither the proposed probabilistic model nor the overall methodology related to the identification through a statistical inverse problem.

\subsubsection{Virtual mechanical testing in MD simulations}

In order to obtain independent realizations of the apparent stiffness properties of the nanocomposite with $n_{\mathrm{pc}}=10$ (see $[49,30]$ ), each configuration is submitted to a mechanical loading under NPT conditions, with a target pressure tensor of the following form:

$$
P^{\alpha \beta}(t)_{i j}=\frac{p^{\alpha \beta}(t)}{2}\left(\delta_{i \alpha} \delta_{j \beta}+\delta_{i \beta} \delta_{j \alpha}\right),
$$

where $\alpha$ and $\beta$ are fixed integers in $\{1,2,3\}$, and $t \mapsto p^{\alpha \beta}(t)$ is a stepwise function corresponding to a quasi-static discretization of a linear loading function (with a loading rate equal to 1 bar per 2 ps) between the initial and final pressures - hence limiting viscosity effects in the polymer system. For a given initial configuration, average deformations of the MD box are then extracted all along the loading and used to compute, by means of an ergodic estimator, the associated realization of the compliance tensor. Because of the roughness of small-diameter inclusions, six different loading conditions are sequentially applied in order to recover the whole slightly anisotropic tensor. These 
conditions correspond to the choices $1 \leqslant \alpha \leqslant 3$ and $\alpha \leqslant \beta$ in Eq. (13). The deformations of the MD box for $\alpha=\beta=1$ and $\{\alpha=1, \beta=2\}$ are shown in Figs. 6 and 7, respectively. The stress-strain curves for both the pure polymer and the nanocomposite system are shown in Fig. 8 (here, ergodic estimators for the components of the stress and strain tensors are computed at each load increment, hence resulting in smoothed stress-strain curves), for the above loading cases. As
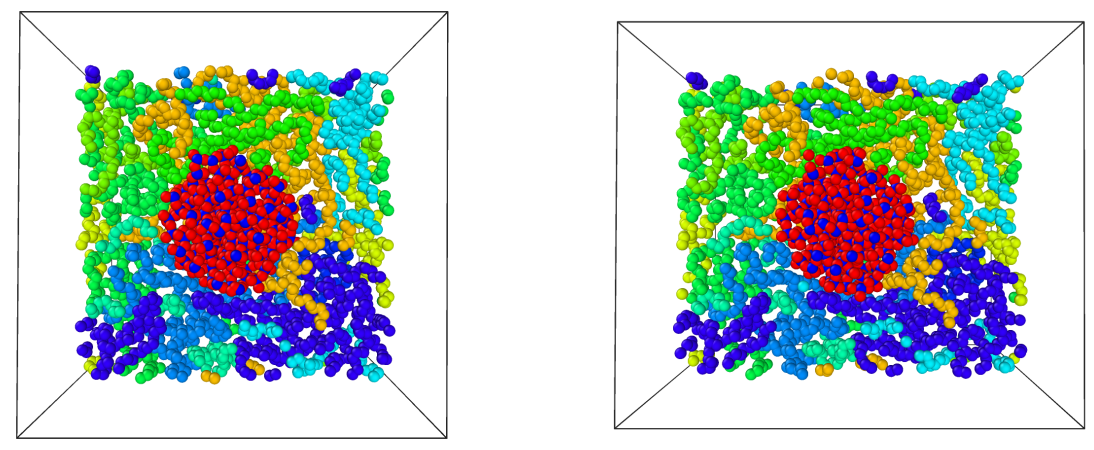

Figure 6: Deformation of the MD box for the loading $t \mapsto\left[P^{11}(t)\right]$ (tensile test): initial configuration (left) and configuration deformed at $10 \%$ (right).
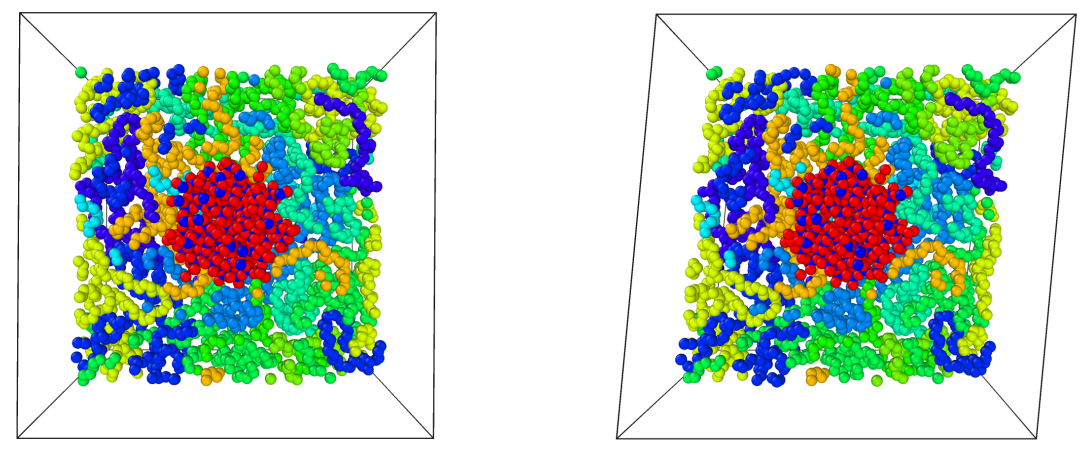

Figure 7: Deformation of the MD box for the loading $t \mapsto\left[P^{12}(t)\right]$ (shear loading): initial configuration (left) and configuration deformed at $5 \%$ (right).

expected, it is seen that the nanocomposite system exhibits a stiffer mechanical behavior than the polymer matrix. 

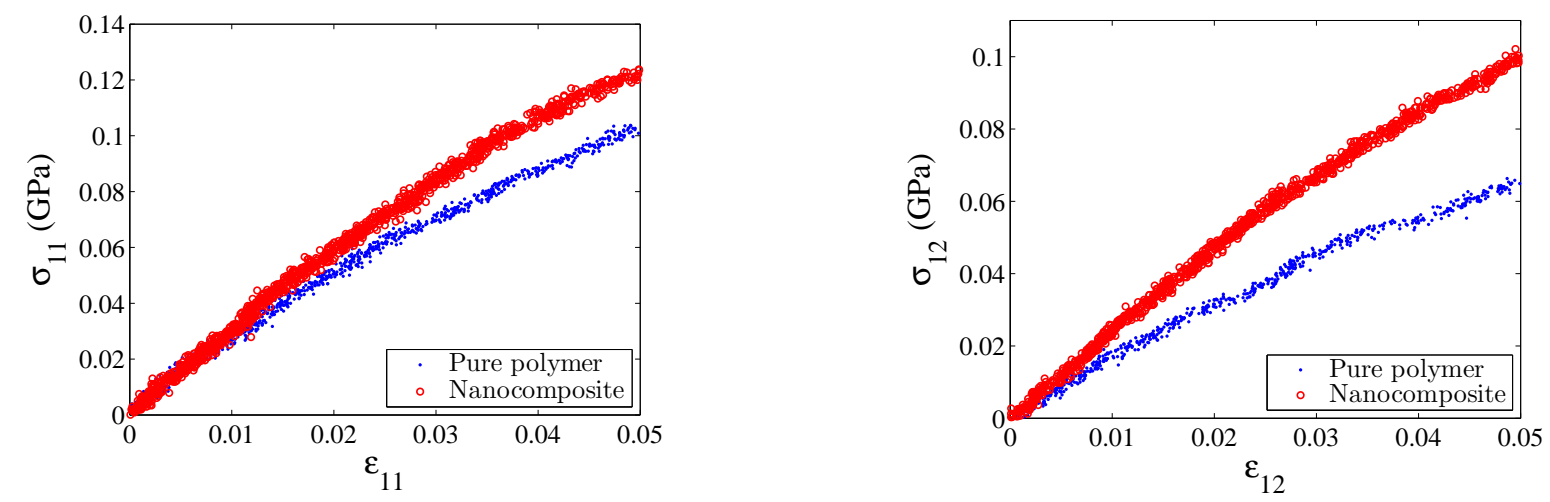

Figure 8: Stress-strain curves simulated for the pure polymer and the nanocomposite: tensile test (left) and shear loading (right).

\section{Stochastic modeling of the elasticity random field in the interphase region}

In this section, we address the probabilistic modeling of the elasticity random field in the interphase region, which is denoted by $\mathcal{D}_{\mathcal{I}}$. Let $\left(O, \boldsymbol{e}^{r}, \boldsymbol{e}^{\theta}, \boldsymbol{e}^{\varphi}\right)$ be the system of spherical coordinates, and denote by $\boldsymbol{x}^{s}=(r, \theta, \varphi)$ any generic point of $\mathbb{R}^{3}$ expressed in this coordinate system. Let $\boldsymbol{x}=\left(x_{1}, x_{2}, x_{3}\right)$ denote any point of $\mathbb{R}^{3}$ given in the cartesian coordinate system, with $x_{1}=$ $r \sin (\varphi) \cos (\theta), x_{2}=r \sin (\varphi) \sin (\theta)$ and $x_{3}=r \cos (\varphi)$. From the results of the MD simulations, it is postulated that:

- the interphase region can be represented as a spherical shell of constant thickness $e_{\mathcal{I}}=2 \mathrm{~nm}$ :

$$
\mathcal{D}_{\mathcal{I}}:=\left\{\boldsymbol{x}^{s}=(r, \theta, \varphi) \mid r \in\left[R_{p}, R_{p}+e_{\mathcal{I}}\right], \theta \in[0,2 \pi], \varphi \in[0, \pi]\right\},
$$

with $R_{p}$ the radius of the particle under consideration (recall that $R_{p}$ is fixed for a given value of $n_{\mathrm{pc}}$ )

- the material in the interphase exhibits a transversely isotropic local behavior defined by the unit normal vector $\boldsymbol{n}\left(\boldsymbol{x}^{s}\right)=\boldsymbol{e}^{r}\left(\boldsymbol{x}^{s}\right)$ at point $\boldsymbol{x}^{s}$;

- the stiffness tensor random field exhibits a mean value that is independent of $\boldsymbol{x}^{s}$ and a correlation structure with a spherical symmetry (in the spherical coordinate system);

- the probabilistic properties of the elasticity random field are similar in the directions defined by $\boldsymbol{e}^{\theta}$ and $\boldsymbol{e}^{\varphi}$.

It is further assumed that the mean model is independent of $\boldsymbol{x}^{s}$ in spherical coordinates. Whereas such a modeling assumption could be readily relaxed by adding, for instance, a dependence along 
the radial direction, it is worth noticing that the model thus obtained would require additional data that are not considered here for model calibration.

\subsection{Overview of the methodology}

Let $\left\{\left[\boldsymbol{C}^{s}\left(\boldsymbol{x}^{s}\right)\right], \boldsymbol{x}^{s} \in \mathcal{D}_{\mathcal{I}}\right\}$ be the random field modeling the stiffness properties in the stochastic interphase. Let $\mathbb{E l a}{ }^{\mathcal{T}}(\boldsymbol{n})$ be the set of transversely isotropic tensors defined by the unit vector $\boldsymbol{n}$. The following algebraic decomposition is first considered:

$$
\forall \boldsymbol{x}^{s} \in \mathcal{D}_{\mathcal{I}}, \quad\left[\boldsymbol{C}^{s}\left(\boldsymbol{x}^{s}\right)\right]=\eta\left[I_{6}\right]+\left[\boldsymbol{\mathcal { M }}^{s}\left(\boldsymbol{x}^{s}\right)\right],
$$

where $\eta \ll 1$ is an arbitrary small parameter, $\left[I_{6}\right]$ is the $6-b y-6$ identity matrix and $\left\{\left[\mathcal{M}^{s}\left(\boldsymbol{x}^{s}\right)\right], \boldsymbol{x}^{s} \in\right.$ $\left.\mathcal{D}_{\mathcal{I}}\right\}$ is a random field with values in $\mathbb{E} \mathrm{la}^{\mathcal{T} \mathcal{I}}(\boldsymbol{n})$, with $\boldsymbol{n}=(1,0,0)$ in spherical coordinates. The first term in the right-hand side of Eq. (47) is introduced in order to preserve the uniform ellipticity of the stochastic boundary value problem. Next, for all $\boldsymbol{x}^{s}$ fixed in $\mathcal{D}_{\mathcal{I}}$, the random matrix $\left[\mathcal{M}^{s}\left(\boldsymbol{x}^{s}\right)\right]$ is normalized according to

$$
\left[\mathcal{M}^{s}\left(\boldsymbol{x}^{s}\right)\right]=\left[\underline{\mathcal{M}}^{s}\right]^{1 / 2}\left[\mathcal{N}^{s}\left(\boldsymbol{x}^{s}\right)\right]\left[\underline{\mathcal{M}}^{s}\right]^{1 / 2}, \quad \forall \boldsymbol{x}^{s} \in \mathcal{D}_{\mathcal{I}},
$$

where $\underline{\mathcal{M}}^{s}=\mathbb{E}\left\{\left[\boldsymbol{C}^{s}\left(\boldsymbol{x}^{s}\right)\right]\right\}-\eta\left[I_{6}\right]$ (note that the mean function depends on $\boldsymbol{x}$ in the cartesian system), and $\left\{\left[\mathcal{N}^{s}\left(\boldsymbol{x}^{s}\right)\right], \boldsymbol{x}^{s} \in \mathcal{D}_{\mathcal{I}}\right\}$ is a random field such that $\mathbb{E}\left\{\left[\mathcal{N}^{s}\left(\boldsymbol{x}^{s}\right)\right]\right\}=\left[I_{6}\right], \forall \boldsymbol{x}^{s} \in \mathcal{D}_{\mathcal{I}}$. It can then be shown (see [50]) that there exists a unique random field $\left\{\left[\mathcal{G}^{s}\left(\boldsymbol{x}^{s}\right)\right], \boldsymbol{x}^{s} \in \mathcal{D}_{\mathcal{I}}\right\}$ such that

$$
\forall \boldsymbol{x}^{s} \in \mathcal{D}_{\mathcal{I}}, \quad\left[\mathcal{N}^{s}\left(\boldsymbol{x}^{s}\right)\right]=\exp \left\{\left[\mathcal{G}^{s}\left(\boldsymbol{x}^{s}\right)\right]\right\}
$$

with

$$
\left[\mathcal{G}^{s}\left(\boldsymbol{x}^{s}\right)\right]=\sum_{i=1}^{5} G_{i}^{s}\left(\boldsymbol{x}^{s}\right)\left[E^{i}\right],
$$

where $\left\{G_{i}^{s}\left(\boldsymbol{x}^{s}\right), \boldsymbol{x}^{s} \in \mathcal{D}_{\mathcal{I}}\right\}, i \in\{1, \ldots, 5\}$, are non-Gaussian dependent $\mathbb{R}$-valued random fields and $\left\{\left[E^{i}\right]\right\}_{i=1}^{5}$ denotes the matrix form of the so-called Walpole basis [51] of $\mathbb{E l a}^{\mathcal{T} \mathcal{I}}(\boldsymbol{n})$, with $\boldsymbol{n}=(1,0,0)$. Let $\left\{\boldsymbol{G}^{s}\left(\boldsymbol{x}^{s}\right), \boldsymbol{x}^{s} \in \mathcal{D}_{\mathcal{I}}\right\}$ be the $\mathbb{R}^{5}$-valued random field such that $\boldsymbol{G}^{s}\left(\boldsymbol{x}^{s}\right)=\left(G_{1}^{s}\left(\boldsymbol{x}^{s}\right), \ldots, G_{5}^{s}\left(\boldsymbol{x}^{s}\right)\right)$. Following [52, 50], the probabilistic model for random field $\left\{\boldsymbol{G}\left(\boldsymbol{x}^{s}\right), \boldsymbol{x}^{s} \in \mathcal{D}_{\mathcal{I}}\right\}$ is constructed by following a two-step strategy:

- first, the first-order marginal probability density function of the random field is constructed in the context of Information Theory [53] and more specifically, by invoking the principle of maximum entropy $[53,54]$ (with information implied by mathematical properties or inferred from atomistic simulations);

- second, the non-Gaussian vector-valued random field $\left\{\boldsymbol{G}^{s}\left(\boldsymbol{x}^{s}\right), \boldsymbol{x}^{s} \in \mathcal{D}_{\mathcal{I}}\right\}$ is defined through a nonlinear mapping acting on an underlying centered second-order $\mathbb{R}^{5}$-valued Gaussian random field, the parametrized correlation structure of which is transported to $\left\{\boldsymbol{G}^{s}\left(\boldsymbol{x}^{s}\right), \boldsymbol{x}^{s} \in \mathcal{D}_{\mathcal{I}}\right\}$. 


\subsection{Construction of the family of first-order marginal probability distributions}

The maximum entropy principle is formulated by considering a constraint on the mean value (raising from Eq. (16)),

$$
\mathbb{E}\left\{\exp \left(\sum_{i=1}^{5} G_{i}^{s}\left(\boldsymbol{x}^{s}\right)\left[E^{i}\right]\right)\right\}=\left[I_{6}\right],
$$

as well as a constraint related to the finiteness of second-order moments for random matrix $\left[\mathcal{N}^{s}\left(\boldsymbol{x}^{s}\right)\right]$ [50] (see [55]):

$$
\sum_{i=1}^{5} \mathbb{E}\left\{G_{i}^{s}\left(\boldsymbol{x}^{s}\right)\right\} \operatorname{tr}\left(\left[E^{i}\right]\right)=\nu, \quad|\nu|<+\infty,
$$

where $\nu$ is independent of $\boldsymbol{x}^{s}$ following the assumptions stated at the beginning of section 3 . It can be shown that an approximate solution writes

$$
p_{\boldsymbol{G}^{s}\left(\boldsymbol{x}^{s}\right)}(\boldsymbol{g})=c \exp (-\Phi(\boldsymbol{g}))
$$

where $c$ is the normalization constant and the potential function $\Phi$ is defined as [56]

$$
\Phi(\boldsymbol{g})=\alpha \times \delta_{[\mathcal{N}]}^{-2} \times \operatorname{tr}\left(\exp \left(\sum_{i=1}^{5} g_{i}\left[E_{i}\right]\right)-\sum_{i=1}^{5} g_{i}\left[E_{i}\right]\right), \quad \forall \boldsymbol{g} \in \mathbb{R}^{5}
$$

In Eq. (22), $\alpha$ is a parameter which depends on the symmetry under consideration $(\alpha \approx 0.82$ for the set $\mathbb{E}$ la ${ }^{\mathcal{T}}(\boldsymbol{n})$ of transversely isotropic tensors, regardless of the unit normal vector $\left.\boldsymbol{n}\right)$, and $\delta_{[\mathcal{N}]}$ is a scalar parameter independent of $\boldsymbol{x}^{s}$ controlling the level of statistical fluctuations exhibited by $\left[\mathcal{N}^{s}\left(\boldsymbol{x}^{s}\right)\right]$

\subsection{Definition and generator of random field $\left\{\boldsymbol{G}^{s}\left(\boldsymbol{x}^{s}\right), \boldsymbol{x}^{s} \in \mathcal{D}_{\mathcal{I}}\right\}$}

\subsubsection{Definition of a $\boldsymbol{x}^{s}$-dependent family of Wiener processes}

Let $\left\{\boldsymbol{\Xi}\left(\boldsymbol{x}^{s}\right)=\left(\xi^{1}\left(\boldsymbol{x}^{s}\right), \ldots, \xi^{5}\left(\boldsymbol{x}^{s}\right)\right), \boldsymbol{x}^{s} \in \mathcal{D}_{\mathcal{I}}\right\}$ be the centered Gaussian $\mathbb{R}^{5}$-valued random field with independent components that are defined as follows. The diagonal entries of the continuous correlation function $\left(\boldsymbol{x}^{s}, \boldsymbol{y}^{s}\right) \mapsto\left[\mathrm{R}_{\Xi}\left(\boldsymbol{x}^{s}, \boldsymbol{y}^{s}\right)\right]$ with values in the set of diagonal real matrices are such that

$$
\left[\mathrm{R}_{\boldsymbol{\Xi}}\left(\boldsymbol{x}^{s}, \boldsymbol{y}^{s}\right)\right]_{i i}=\mathbb{E}\left\{\xi^{i}\left(\boldsymbol{y}^{s}\right) \xi^{i}\left(\boldsymbol{x}^{s}\right)\right\}=: \rho\left(\boldsymbol{x}^{s}, \boldsymbol{y}^{s}\right)
$$

with

$$
\left[\mathrm{R}_{\Xi}\left(\boldsymbol{x}^{s}, \boldsymbol{x}^{s}\right)\right]_{i i}=1, \quad 1 \leqslant i \leqslant 5
$$

for all $\left(\boldsymbol{x}^{s}, \boldsymbol{y}^{s}\right)$ in $\mathcal{D}_{\mathcal{I}} \times \mathcal{D}_{\mathcal{I}}$. It follows that the correlation structure is completely defined by the normalized correlation function $\rho$. A separable structure is further assumed and written, for all $\boldsymbol{x}^{s}=(r, \theta, \varphi)$ and $\boldsymbol{y}^{s}=\left(r^{\prime}, \theta^{\prime}, \varphi^{\prime}\right)$ in $\mathcal{D}_{\mathcal{I}}$, as:

$$
\forall\left(\boldsymbol{x}^{s}, \boldsymbol{y}^{s}\right) \in \mathcal{D}_{\mathcal{I}} \times \mathcal{D}_{\mathcal{I}}, \quad \rho\left(\boldsymbol{x}^{s}, \boldsymbol{y}^{s}\right):=\rho_{r}\left(\tau_{r}\right) \times \rho_{\theta}\left(r, r^{\prime}, \tau_{\theta}\right) \times \rho_{\varphi}\left(r, r^{\prime}, \tau_{\varphi}\right),
$$


where $\tau_{r}:=r-r^{\prime}, \tau_{\theta}:=\theta-\theta^{\prime}$ and $\tau_{\varphi}:=\varphi-\varphi^{\prime}$ are the radial and angular lags. Here, the above correlation functions are parametrized as:

$$
\left\{\begin{array}{l}
\rho_{r}\left(\tau_{r}\right):=\left(\frac{2 L_{r}}{\pi \tau_{r}}\right)^{2} \sin ^{2}\left(\frac{\pi \tau_{r}}{2 L_{r}}\right), \\
\rho_{\theta}\left(r, r^{\prime}, \tau_{\theta}\right):=\exp \left(-\frac{1}{2}\left(\frac{r+r^{\prime}}{L_{\theta}}\right)^{2} \sin ^{2}\left(\frac{\tau_{\theta}}{2}\right)\right), \\
\rho_{\varphi}\left(r, r^{\prime}, \tau_{\varphi}\right):=\exp \left(-\frac{1}{2}\left(\frac{r+r^{\prime}}{L_{\varphi}}\right)^{2} \sin ^{2}\left(\frac{\tau_{\varphi}}{2}\right)\right),
\end{array}\right.
$$

where $L_{r}$ is the spatial correlation length along the radial direction and $\left(L_{\theta}, L_{\varphi}\right)$ is a set of parameters controlling the decorrelation rates along the directions defined by $\boldsymbol{e}^{\theta}$ and $\boldsymbol{e}^{\varphi}$. For given values of $r$ and $r^{\prime}$ in $\left[0, R_{p}\right]$, the functions $\rho_{\theta}$ and $\rho_{\varphi}$ are $2 \pi$-periodic and satisfy:

- $\rho_{\theta}\left(r, r^{\prime},-\tau_{\theta}\right)=\rho_{\theta}\left(r, r^{\prime}, \tau_{\theta}\right)$ for all $\tau_{\theta}$ in $[-2 \pi, 2 \pi]$;

- $\rho_{\varphi}\left(r, r^{\prime},-\tau_{\varphi}\right)=\rho_{\varphi}\left(r, r^{\prime}, \tau_{\varphi}\right)$ for all $\tau_{\varphi}$ in $[-\pi, \pi]$.

Note that the correlation functions $\rho_{r}, \rho_{\theta}$ and $\rho_{\varphi}$ could be parametrized by using other algebraic forms satisfying the conditions rising from the spherical description of the correlation structure. Similarly, the angular correlation functions may be represented by means of expansions in Fourier series. However, the above forms interestingly depend on a small number of parameters and are thus well suited for an inverse identification from limited data.

Let $\boldsymbol{W}=\left\{\boldsymbol{W}\left(t, \boldsymbol{x}^{s}\right), t \geqslant 0, \boldsymbol{x}^{s} \in \mathcal{D}_{\mathcal{I}}\right\}$ be the second-order centered Gaussian $\mathbb{R}^{5}$-valued random field defined as follows:

- $\forall \boldsymbol{x}^{s} \in \mathcal{D}_{\mathcal{I}}, \boldsymbol{W}\left(0, \boldsymbol{x}^{s}\right)=\mathbf{0}$ almost surely;

- the generalized derivative $\mathrm{D}_{t} \boldsymbol{W}$ of $\boldsymbol{W}$ (with respect to $t$ ) is the normalized Gaussian cylindrical white noise $\boldsymbol{B}$ whose covariance function $\left[\mathrm{C}_{\boldsymbol{B}}\right]$ writes, for all $\forall\left(\boldsymbol{x}^{s}, \boldsymbol{y}^{s}\right) \in \mathcal{D}_{\mathcal{I}} \times \mathcal{D}_{\mathcal{I}}$ and for all $\tau \in \mathbb{R}$ :

$$
\left[\mathrm{C}_{\boldsymbol{B}}\left(\boldsymbol{x}^{s}, \boldsymbol{y}^{s}, t+\tau, t\right)\right]:=\delta_{0}(\tau)\left[\mathrm{R}_{\Xi}\left(\boldsymbol{x}^{s}, \boldsymbol{y}^{s}\right)\right]
$$

with $\delta_{0}$ the generalized Delta function at 0 .

For any $\boldsymbol{x}^{s}$ fixed in $\mathcal{D}_{\mathcal{I}}$, the stochastic process $\{\boldsymbol{W}(t, \boldsymbol{x}), t \geqslant 0\}$ is then a normalized $\mathbb{R}^{5}$-valued Wiener process (note that variable $t$ does not have to be confused with time variable and in particular, with the time variable involved in the atomistic simulations). 


\subsubsection{Random generator}

Let $\boldsymbol{x}^{s}$ be fixed in $\mathcal{D}_{\mathcal{I}}$. Let $\left\{\left(\boldsymbol{U}\left(r, \boldsymbol{x}^{s}\right), \boldsymbol{V}\left(r, \boldsymbol{x}^{s}\right)\right), t \geqslant 0\right\}$ be the $\mathbb{R}^{5} \times \mathbb{R}^{5}$-valued Markov process satisfying the following Itô stochastic differential equation [50]:

$$
\left\{\begin{array}{l}
\mathrm{d} \boldsymbol{U}\left(t, \boldsymbol{x}^{s}\right)=\boldsymbol{V}\left(t, \boldsymbol{x}^{s}\right) \mathrm{d} t \\
\mathrm{~d} \boldsymbol{V}\left(t, \boldsymbol{x}^{s}\right)=\left(-\nabla_{\boldsymbol{u}} \Phi\left(\boldsymbol{U}\left(t, \boldsymbol{x}^{s}\right)\right)-\frac{\varrho}{2} \boldsymbol{V}\left(t, \boldsymbol{x}^{s}\right)\right) \mathrm{d} t+\sqrt{\eta} \mathrm{d} \boldsymbol{W}\left(t, \boldsymbol{x}^{s}\right)
\end{array}\right.
$$

for all $t \in \mathbb{R}^{+}$, where $\Phi$ is the potential function defined by Eq. (22), $\left.\varrho \in\right] 0,+\infty[$ is a tunable parameter and $\left\{\boldsymbol{W}\left(t, \boldsymbol{x}^{s}\right), t \geqslant 0\right\}$ is the Wiener process defined above. Initial conditions are written as $\boldsymbol{U}\left(0, \boldsymbol{x}^{s}\right)=\boldsymbol{U}^{0}\left(\boldsymbol{x}^{s}\right)$ and $\boldsymbol{V}\left(0, \boldsymbol{x}^{s}\right)=\boldsymbol{V}^{0}\left(\boldsymbol{x}^{s}\right)$ almost surely, where the probability distribution of random variable $\left(\boldsymbol{U}^{0}\left(\boldsymbol{x}^{s}\right), \boldsymbol{V}^{0}\left(\boldsymbol{x}^{s}\right)\right)$ is assumed to be given. It can then be shown that [57]

$$
\lim _{t \rightarrow+\infty} \boldsymbol{U}\left(t, \boldsymbol{x}^{s}\right) \stackrel{\operatorname{law}}{=} \boldsymbol{G}^{s}\left(\boldsymbol{x}^{s}\right)
$$

hence providing a sampling algorithm for the non-Gaussian random field $\left\{\boldsymbol{G}^{s}\left(\boldsymbol{x}^{s}\right), \boldsymbol{x}^{s} \in \mathcal{D}_{\mathcal{I}}\right\}$. In this work, the stochastic differential equation defined by Eq. (28) is discretized by means of a Störmer-Verlet algorithm [58, 59, 60, 61] (see e.g. [62, 63, 64] for thorough reviews on discretization schemes).

\section{Inverse identification of the random field model from atomistic computations}

\subsection{Overall methodology}

Let $\left\{\left[\widetilde{C}_{\mathrm{MD}}\left(\omega_{i}\right)\right]\right\}_{i=1}^{20}$ be the set of realizations for the apparent stiffness tensor estimated from MD simulations, which is denoted by $\left[\widetilde{\boldsymbol{C}}_{\mathrm{MD}}\right]$ (see section 2.4.2). From the previous section, one has

$$
\forall \boldsymbol{x}^{s} \in \mathcal{D}_{\mathcal{I}}, \quad\left[\boldsymbol{C}^{s}\left(\boldsymbol{x}^{s}\right)\right]=\eta\left[I_{6}\right]+\left[\underline{\mathcal{M}}^{s}\right]^{1 / 2} \exp \left\{\left[\mathcal{G}^{s}\left(\boldsymbol{x}^{s}\right)\right]\right\}\left[\underline{\mathcal{M}}^{s}\right]^{1 / 2},
$$

so that the probabilistic model for random field $\left\{\boldsymbol{C}^{s}\left(\boldsymbol{x}^{s}\right), \boldsymbol{x}^{s} \in \mathcal{D}_{\mathcal{I}}\right\}$ depends on two vectors of hyperparameters, namely:

- a vector $\boldsymbol{h}_{1}$ of hyperparameters gathering the coordinates of mean value $\left[\underline{\mathcal{M}}^{s}\right]$ onto matrix basis $\left\{\left[E^{i}\right]\right\}_{i=1}^{5}$, with $\boldsymbol{h}_{1}=\left(\underline{m}_{1}, \ldots, \underline{m}_{5}\right) \in \mathcal{S}_{1} \subset \mathbb{R}^{5}$;

- a vector $\boldsymbol{h}_{2}=\left(\delta_{[\mathcal{N}]}, L_{r}, L_{a}\right) \in \mathcal{S}_{2}$ of hyperparameters related to the level of fluctuations and to the correlation functions, where it is assumed (based from the MD results) that $L_{\theta}=L_{\varphi}=: L_{a}$. 
In this work, the optimal values of the above vectors, denoted by $\boldsymbol{h}_{1}^{\text {opt }}$ and $\boldsymbol{h}_{2}^{\text {opt }}$ respectively, are identified by stating the equivalence between the apparent properties obtained from MD simulations (with $n_{\mathrm{pc}}=10$ ) and those determined by using standard computational homogenization in a continuum mechanics (CM) formulation. It should be noted that realizations of the local stiffness tensor field cannot be extracted from MD simulations and readily passed into the CM formulation (hence, a calibration based on a statistical inverse problem), for at least two reasons. First, the definition and CM-based interpretation of local properties extracted from MD results (see [65, 66]) is still an open question whenever confined matter is involved [30]. Second, the loading conditions applied in the atomistic virtual testing (where the local properties are triclinic with respect to the MD reference frame) are very different from those considered in the continuum setting, so that imposing a match, in some probabilistic sense, between the two local fields may not yield reliable results.

Let us now introduce the continuum model. Let $\mathcal{D}$ be the three-dimensional open bounded domain in $\mathbb{R}^{3}$ such that $\mathcal{D}=(]-L_{\mathcal{D}} / 2, L_{\mathcal{D}} / 2[)^{3}$, with $L_{\mathcal{D}}=6.68(\mathrm{~nm})$, and denote by $\partial \mathcal{D}$ its boundary. Below, $\mathcal{B}_{O}(r)$ denotes the ball of radius $r$ (in $\mathrm{nm}$ ), centered at the origin $O$ of the cartesian reference frame $\left(O, \boldsymbol{e}^{1}, \boldsymbol{e}^{2}, \boldsymbol{e}^{3}\right)$. The Silica nanoparticle is then modeled by the ball $\mathcal{D}_{\text {Silica }}:=\mathcal{B}_{O}(1.5)$, whereas the interphase region is represented by domain $\mathcal{D}_{\mathcal{I}}:=\mathcal{D} \cap\left(\mathcal{B}_{O}(3.5) \backslash \mathcal{B}_{O}(1.5)\right)$. It is seen that the diameter of the ball $\mathcal{B}_{O}(3.5)$ is slightly larger than the characteristic length of $\mathcal{D}$, but this configuration has a negligible effect in the computational results provided below. The domain occupied by the pure polymer bulk is thus $\mathcal{D}_{\mathcal{P}}:=\mathcal{D} \backslash\left(\mathcal{D}_{\text {Silica }} \cup \mathcal{D}_{\mathcal{I}}\right)$.

The properties of each deterministic isotropic phase were estimated from MD simulations and are as follows ( $k$ and $\mu$ denote the bulk and shear moduli, respectively):

- for the Silica inclusion: $k_{\text {Silica }}=36.8, \mu_{\text {Silica }}=30.5$ (both in GPa).

- for the polymer bulk: $k_{\mathrm{pbk}}=5.19, \mu_{\mathrm{pbk}}=0.93$ (both in GPa).

In the interphase region, the elastic properties are modeled by the random field model (detailed in section 3) that is described, from now on, by using the cartesian coordinate system - hence, the omission of superscript "s".

\subsubsection{Homogenization problem for the continuum mechanics modeling}

In order to formulate the statistical inverse problem, let us now introduce the upscaling procedure in the continuum mechanics framework. This procedure is based on averaging procedures that are widely used in homogenization theories. However, and as opposed to classical assumptions stated in such theories, it is worthwhile to notice that $\mathcal{D}$ is not assumed to be a representative volume element, so that upscaled properties are coined as apparent ones [67] and exhibit non-negligible statistical fluctuations. 
For selected values of $\boldsymbol{h}_{1}$ and $\boldsymbol{h}_{2}$, let us consider the following stochastic boundary value problem (SBVP) [68]:

$$
\begin{aligned}
-\operatorname{div}[\boldsymbol{\sigma}(\boldsymbol{x})]=0 & , \quad \forall \boldsymbol{x} \in \mathcal{D}, \\
{[\boldsymbol{\sigma}(\boldsymbol{x})] \boldsymbol{n}(\boldsymbol{x})=[\Sigma] \boldsymbol{n}(\boldsymbol{x}) } & , \quad \forall \boldsymbol{x} \in \partial \mathcal{D},
\end{aligned}
$$

where $\boldsymbol{x} \mapsto[\boldsymbol{\sigma}(\boldsymbol{x})]$ is the local stress field, $\boldsymbol{n}(\boldsymbol{x})$ is the unit vector normal to $\partial \mathcal{D}$ at point $\boldsymbol{x}$, and $[\Sigma]$ is the macroscopic stress tensor. The constitutive law writes:

$$
[\boldsymbol{\sigma}(\boldsymbol{x})]=\llbracket \boldsymbol{C}(\boldsymbol{x}) \rrbracket:[\boldsymbol{\epsilon}(\boldsymbol{x})], \quad \forall \boldsymbol{x} \in \mathcal{D},
$$

where $\llbracket \boldsymbol{C}(\boldsymbol{x}) \rrbracket$ is the fourth-order tensor representation of elasticity matrix $[\boldsymbol{C}(\boldsymbol{x})]$. Note that $\llbracket \boldsymbol{C}(\boldsymbol{x}) \rrbracket$ is random whenever $\boldsymbol{x} \in \mathcal{D}_{\mathcal{I}}$, and deterministic elsewhere (here, and for the sake of notational convenience, the same notation is used regardless of the deterministic or random nature of the elasticity tensor at a given point). It can then be shown that there exists a so-called stress concentration tensor field $\boldsymbol{x} \mapsto \llbracket \boldsymbol{B}(\boldsymbol{x}) \rrbracket$ with values in the set of fourth-order tensors (with minor symmetries) such that

$$
[\boldsymbol{\sigma}(\boldsymbol{x})]=\llbracket \boldsymbol{B}(\boldsymbol{x}) \rrbracket:[\Sigma], \quad \forall \boldsymbol{x} \in \mathcal{D}
$$

and

$$
\frac{1}{|\mathcal{D}|} \int_{\mathcal{D}} \llbracket \boldsymbol{B}(\boldsymbol{x}) \rrbracket \mathrm{d} \boldsymbol{x}=\llbracket I \rrbracket
$$

with $\llbracket I \rrbracket$ the fourth-order symmetric identity tensor. By construction, one has

$$
B_{i j k \ell}(\boldsymbol{x})=\sigma_{i j}^{k \ell}(\boldsymbol{x}),
$$

where $\boldsymbol{x} \mapsto\left[\boldsymbol{\sigma}^{k \ell}(\boldsymbol{x})\right]$ is the stress field solving the aforementioned stochastic boundary value problem with the macroscopic stress tensor $\Sigma^{k \ell}$ given by:

$$
\Sigma_{i j}^{k \ell}=\frac{1}{2}\left(\delta_{i k} \delta_{j \ell}+\delta_{i \ell} \delta_{j k}\right)
$$

It follows that the random field $\boldsymbol{x} \mapsto \llbracket \boldsymbol{B}(\boldsymbol{x}) \rrbracket$ can be recovered by solving the above stochastic boundary value problem for six different values of the macroscopic stress tensor $\Sigma^{k \ell}$, with $k$ and $\ell$ in $\{1,2,3\}$. The apparent compliance tensor $\llbracket \widetilde{\boldsymbol{S}} \rrbracket$ defined with respect to the statically uniform boundary conditions is then defined as:

$$
\llbracket \widetilde{\boldsymbol{S}} \rrbracket=\frac{1}{|\mathcal{D}|} \int_{\mathcal{D}} \llbracket \boldsymbol{S}(\boldsymbol{x}) \rrbracket: \llbracket \boldsymbol{B}(\boldsymbol{x}) \rrbracket \mathrm{d} \boldsymbol{x},
$$

where $\boldsymbol{x} \mapsto \llbracket \boldsymbol{S}(\boldsymbol{x}) \rrbracket$ is the local fourth-order compliance tensor field. Let $\llbracket \widetilde{\boldsymbol{C}} \rrbracket=\llbracket \widetilde{\boldsymbol{S}} \rrbracket^{-1}$ be the associated apparent stiffness tensor, and denote by $\left[\widetilde{\boldsymbol{C}}_{\mathrm{CM}}\left(\boldsymbol{h}_{1}, \boldsymbol{h}_{2}\right)\right]$ its second-order tensor representation 
(where the dependence on $\boldsymbol{h}_{1}$ and $\boldsymbol{h}_{2}$ is made explicit for subsequent use). The above apparent tensors depend on the applied boundary conditions (as discussed in the literature [69]), which are chosen in accordance with the boundary conditions that are considered in the MD simulations (it is worth recalling here that the virtual testing is carried out by prescribing a target macroscopic pressure tensor in the NPT ensemble). Similarly, $\left[\widetilde{C}_{\mathrm{CM}}\left(\boldsymbol{h}_{1}\right)\right]$ denotes the apparent tensor (under the same boundary conditions) obtained by considering an interphase with deterministic properties defined by vector $\boldsymbol{h}_{1}$ in spherical coordinates.

In this work, the mechanical problem defined by Eqs. (31) and (34) is solved, for any realization of the random field in the interphase region, by the finite element method. Domain $\mathcal{D}$ is discretized with 4-node tetrahedron elements (with 1 Gauss integration point) - see Fig. 9. The final computational model is made up of 190, 310 elements (corresponding to 102,561 degrees of freedom). It
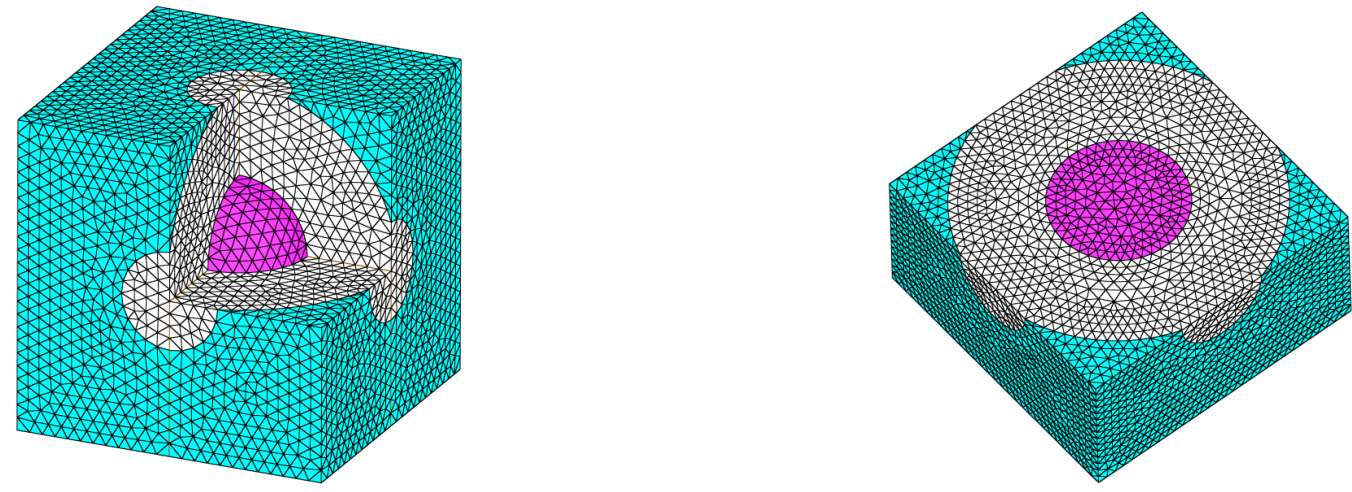

Figure 9: 3D views of the meshed continuum model (the inclusion appears in magenta, the interphase in white and the polymer matrix in turquoise).

is worth noticing that such a mesh density provides at least four integration points per correlation length (in mean), regardless of the direction or the configuration tested while solving the statistical inverse problem, hence ensuring a good sampling of the correlation structure.

Remark on random field sampling by the ergodic method. Assume that each ISDE is solved by using a discretization depending on step $\Delta t$ for parameter $t$, and denote by $t^{*}:=\left(k^{*}-1\right) \Delta t$ the first instance of $t$ within the stationary regime. For $1 \leqslant i \leqslant j \leqslant 5$, a decorrelation index $\tau_{i j}^{\varepsilon}\left(\boldsymbol{x}^{s}\right)$ associated with stochastic processes $\left\{U_{i}\left(t, \boldsymbol{x}^{s}\right), t \geqslant t^{*}\right\}$ and $\left\{U_{j}\left(t, \boldsymbol{x}^{s}\right), t \geqslant t^{*}\right\}$ (where $\boldsymbol{x}^{s}$ is any point in $\left.\mathcal{D}_{\mathcal{I}}\right)$ is then introduced and such that

$$
\mathbb{E}\left\{U_{j}\left(\left(k+\tau_{i j}^{\epsilon}\right) \Delta t, \boldsymbol{x}^{s}\right) U_{i}\left(k \Delta t, \boldsymbol{x}^{s}\right)\right\} \leqslant \varepsilon
$$

for some arbitrary decorrelation threshold $0<\varepsilon \ll 1$ and $k \geqslant k^{*}$. A global decorrelation index $\tau^{\epsilon}$ 
can be defined as

$$
\tau^{\epsilon}=\sup _{\boldsymbol{x}^{s} \in \mathcal{D}_{\mathcal{I}}}\left(\max _{1 \leqslant i \leqslant j \leqslant 5} \tau_{i j}^{\varepsilon}\left(\boldsymbol{x}^{s}\right)\right) .
$$

In practice, and for a sufficiently small value of $\varepsilon \ll 1$, this result means that $\left\{\boldsymbol{U}\left(\left(k+\tau^{\varepsilon}\right) \Delta t, \boldsymbol{x}^{s}\right), \boldsymbol{x}^{s} \in\right.$ $\left.\mathcal{D}_{\mathcal{I}}\right\}$ and $\left\{\boldsymbol{U}\left(k \Delta t, \boldsymbol{x}^{s}\right), \boldsymbol{x}^{s} \in \mathcal{D}_{\mathcal{I}}\right\}, k \geqslant k^{*}$, are weakly correlated samples of $\left\{\boldsymbol{G}^{s}\left(\boldsymbol{x}^{s}\right), \boldsymbol{x}^{s} \in \mathcal{D}_{\mathcal{I}}\right\}$. This sampling strategy is especially relevant whenever the rate of convergence toward the stationary solution at all points in $\mathcal{D}_{\mathcal{I}}$ is low and requires a number of iterations much larger than $\tau^{\varepsilon}$, so that sampling by the Monte Carlo method would require a larger CPU time. The value of parameter $\varepsilon$ must be carefully selected in order to balance sampling quality with computational cost.

\subsubsection{Strategy for model calibration}

The stochastic model is calibrated by solving a statistical inverse problem that is formulated in a two-step manner. First, the vector $\boldsymbol{h}_{1}^{\mathrm{opt}}$ of optimal hyperparameters for the mean model is defined as

$$
\boldsymbol{h}_{1}^{\mathrm{opt}}=\underset{\boldsymbol{h}_{1} \in \mathcal{S}_{1}}{\arg \min } \mathcal{L}_{1}\left(\boldsymbol{h}_{1}\right)
$$

where $\mathcal{L}_{1}$ is the cost function defined as

$$
\mathcal{L}_{1}\left(\boldsymbol{h}_{1}\right):=\frac{\left\|\mathcal{P}^{i s o}\left(\mathbb{E}\left\{\left[\widetilde{\boldsymbol{C}}_{\mathrm{MD}}\right]\right\}\right)-\mathcal{P}^{i s o}\left(\left[\widetilde{C}_{\mathrm{CM}}\left(\boldsymbol{h}_{1}\right)\right]\right)\right\|_{\mathrm{F}}}{\left\|\mathcal{P}^{i s o}\left(\mathbb{E}\left\{\left[\widetilde{\boldsymbol{C}}_{\mathrm{MD}}\right]\right\}\right)\right\|_{\mathrm{F}}},
$$

with $\|\cdot\|_{\mathrm{F}}$ is the Frobenius norm and $\mathcal{S}_{1}$ the admissible set such that the mean model is ensured to be positive-definite. In Eq. (42), $\mathcal{P}^{i s o}$ is the projection operator, defined in the sense of Euclidean norm, on the set of isotropic second-order tensors (see [70] and the references therein). Note that this operator can be shown to commute with the operator of mathematical expectation.

Second, the vector $\boldsymbol{h}_{2}^{\mathrm{opt}}$ of optimal hyperparameters related to both the level of statistical fluctuations and the correlation structure is defined by the maximum likelihood method:

$$
\boldsymbol{h}_{2}^{\text {opt }}=\underset{h_{2} \in \mathcal{S}_{2}}{\arg \max } \mathcal{L}_{2}\left(\boldsymbol{h}_{2}\right)
$$

where $\mathcal{L}_{2}$ the likelihood function given by

$$
\mathcal{L}_{2}\left(\boldsymbol{h}_{2}\right)=\prod_{i=1}^{20} p_{\left[\widetilde{C}_{\mathrm{CM}}\left(\boldsymbol{h}_{1}^{\mathrm{opt}}, \boldsymbol{h}_{2}\right)\right]}\left(\left[\widetilde{C}_{\mathrm{MD}}\left(\omega_{i}\right)\right]\right)
$$

and $p_{\left[\widetilde{\boldsymbol{C}}_{\mathrm{CM}}\left(\boldsymbol{h}_{1}^{\mathrm{opt}}, \boldsymbol{h}_{2}\right)\right]}$ is the probability density function of $\left[\widetilde{\boldsymbol{C}}_{\mathrm{CM}}\left(\boldsymbol{h}_{1}^{\mathrm{opt}}, \boldsymbol{h}_{2}\right)\right]$ estimated by the nonparametric statistics based on the use of the kernel estimation method. 


\subsection{Numerical results}

\subsubsection{Calibration of the mean model}

The optimization defined by Eqs. (41-42) is solved by using the Matlab function fmincon (with default settings) under inequality constraints arising from the definition of $\mathcal{S}_{1}$. The convergence of the algorithm is shown in Fig. 10, where it is seen a good convergence is obtained after 40 iterations.

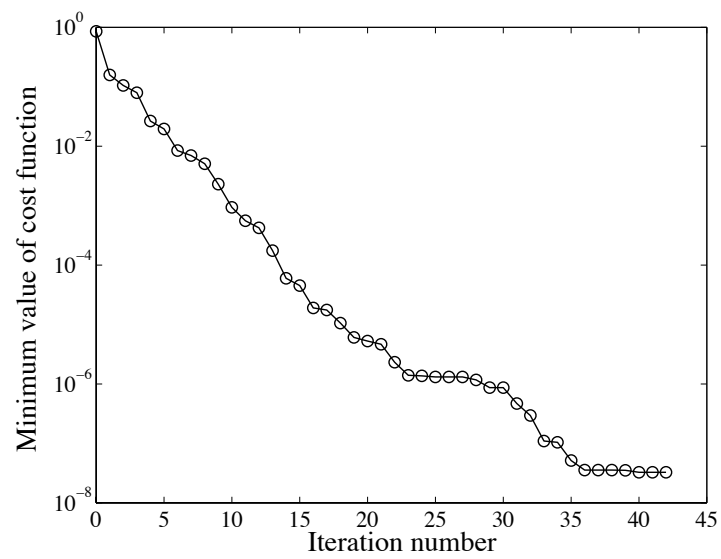

Figure 10: Convergence of the solver for the optimization problem defined by Eqs. (41-42).

The solution $\left[\underline{\mathcal{M}}^{s}\right]=\sum_{i=1}^{5} \underline{m}_{i}^{\text {opt }}\left[E^{i}\right]$ (recall that $\left.\boldsymbol{h}_{1}^{\text {opt }}=\left(\underline{m}_{1}^{\text {opt }}, \ldots, \underline{m}_{5}^{\text {opt }}\right)\right)$ in matrix form is found to be

$$
[\underline{\underline{\mathcal{M}}}]=\left[\begin{array}{cccccc}
5.51 & 4.02 & 4.02 & 0 & 0 & 0 \\
& 5.19 & 1.16 & 0 & 0 & 0 \\
& & 5.19 & 0 & 0 & 0 \\
& & & 4.03 & 0 & 0 \\
& \text { Sym. } & & & 4.25 & 0 \\
& & & & & 4.25
\end{array}\right]
$$

in spherical coordinates (in GPa). A strengthening effect for the shear modulus is thus observed for the mean model in the interphase region (as compared to the bulk properties), which is in accordance with the loss of polymer-chains mobility in the neighborhood of the inclusion, as well as with numerical results obtained elsewhere (see e.g. [71]).

\subsection{Calibration of the level of fluctuations and correlation structure}

Here, the ISDEs are defined with $\varrho=9$ and solved by using a step $\Delta t$ equal to 0.01 (note that these values were obtained from specific convergence analysis). For these parameters, the convergence toward the stationary solution is reached after 20,000 steps. Furthermore, the parameter $\varepsilon$ 
involved in the ergodic sampling is set to 0.1 , which corresponds to a decorrelation index $\tau^{\varepsilon}$ equal to 200. Below, all estimators are computed on a set of 300 realizations obtained from the ergodic sampling introduced at the end of section 4.1.1. The optimization problem defined by Eqs. (43-44) is solved by a regular sampling of the hyperparameters over their admissible sets. The correlation length in the radial direction is normalized by the interphase thickness $e_{\mathcal{I}}$, whereas the parameters related to angular decorrelation are normalized by the term $2 \pi R^{e x t}$, with $R^{e x t}:=R_{p}+e_{\mathcal{I}}$. The plot of the cost function $\mathcal{L}_{2}$ is shown in Fig. 11. The optimal values are found to be $\delta_{[\mathcal{N}]}^{\text {opt }}=0.3$,

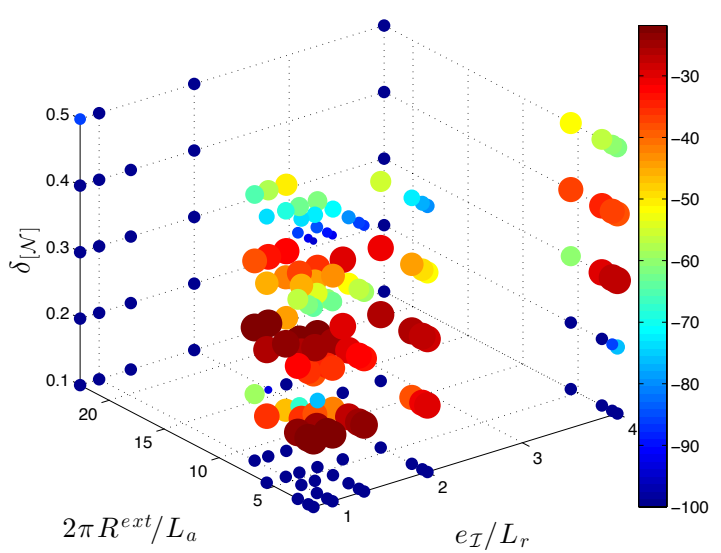

Figure 11: 4D plot of the likelihood function $\boldsymbol{h}_{2} \mapsto \mathcal{L}_{2}\left(\boldsymbol{h}_{2}\right)$ for different combinations of $\delta_{[\mathcal{N}]}, L_{r}$ and $L_{a}$. The values of the cost function are proportional to marker size, as well as to color intensity (infinite values of $\mathcal{L}_{2}$ are arbitrarily replaced by the value -100 for visualization purposes).

$L_{r}^{\mathrm{opt}} \approx 0.9 e_{\mathcal{I}}(=1.8 \mathrm{~nm})$ and $L_{a}^{\mathrm{opt}} \approx 3.85 \mathrm{rad} \times \mathrm{nm}$, hence showing that the fluctuations in the interphase region are neither negligible nor prone to being smoothed out by an upscaling procedure (given that the condition $L_{r}^{\mathrm{opt}} \ll e_{\mathcal{I}}$ is not satisfied for instance). The optimal value of parameter $L_{a}$ further shows that for a given radial position, the elasticity field is substantially correlated over a hemisphere (depending on the distance to particle surface). This result turns out to be consistent with MD results, as qualitatively illustrated in Fig. 12. As an illustration, a realization of the calibrated random field $\left\{C_{11}(\boldsymbol{x}), \boldsymbol{x} \in \partial \mathcal{D}_{\mathcal{I}}^{\text {ext }}\right\}$ (where $\partial \mathcal{D}_{\mathcal{I}}^{\text {ext }}$ denotes the exterior boundary surface of $\mathcal{D}_{\mathcal{I}}$ ) is shown in Fig. 13. Finally, and from the perspective of validation, it is worth investigating whether the continuum can faithfully reproduce, in some sense, the data generated by the MD simulations. In order to get a physical insight on this issue, the realizations obtained from the MD simulations are plotted together with the estimates of (joint) probability density functions for some arbitrarily chosen components of the apparent tensor (defined by the calibrated random field model and through the SBVP stated in section 4.1.1) in Figs. 14 and 15. It is seen that for the selected components, all the realizations obtained by the MD simulations are associated with 

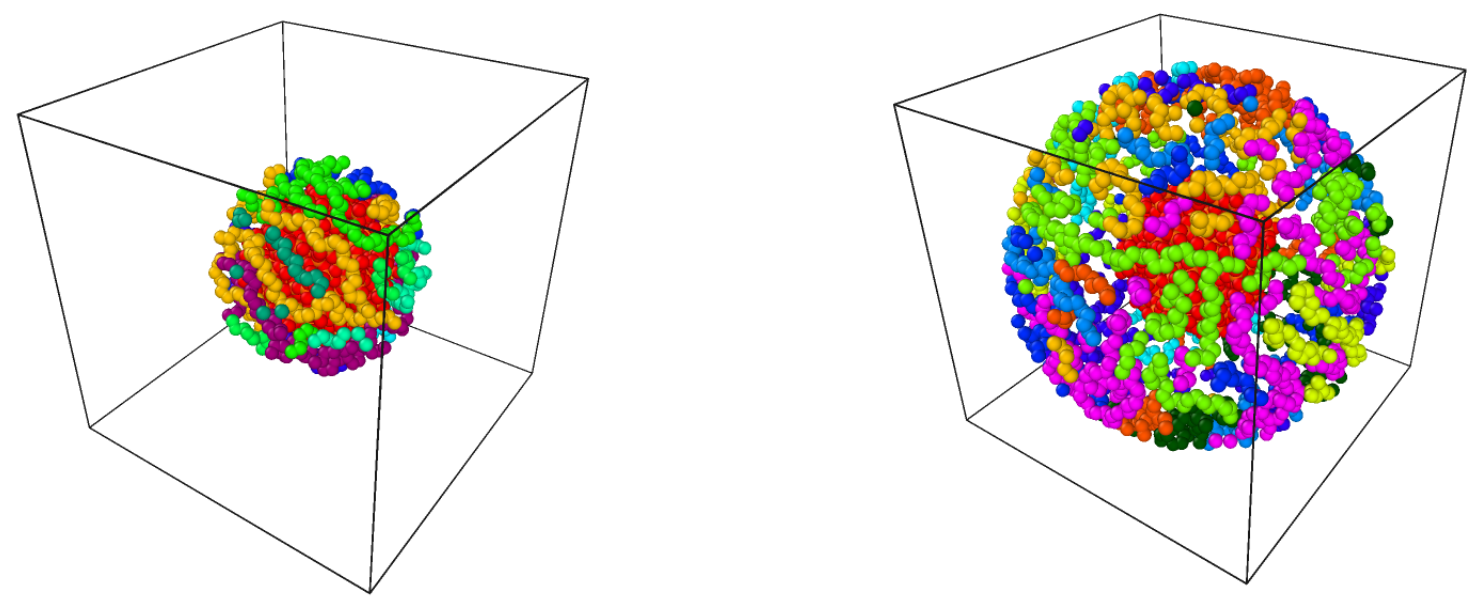

Figure 12: Visualization of an instantaneous configuration of polymer chains trapped into spherical shells of thickness $5 \AA$ within the interphase region. The shell on left (resp. right) panel is the closest (resp. furthest) one to the surface of the nanoparticle.

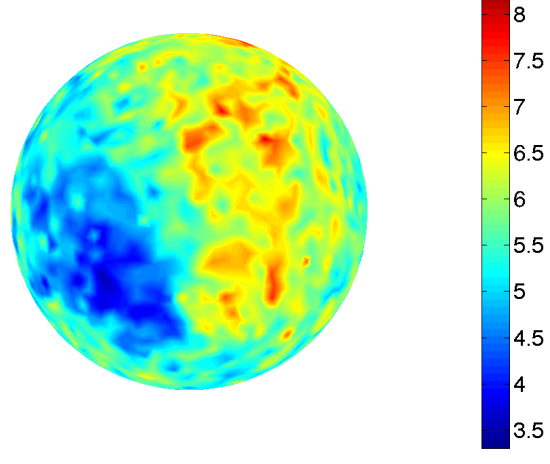

Figure 13: Plot of a realization for elasticity random field $\left\{C_{11}(\boldsymbol{x}), \boldsymbol{x} \in \partial \mathcal{D}_{\mathcal{I}}^{\text {ext }}\right\}$ (in GPa) on the exterior surface of the interphase region (with calibrated hyperparameters).

non-zero probability levels (note that this result holds for all remaining visualizable combinations of components), hence showing in part the consistency of both the probabilistic model and the identification strategy. 

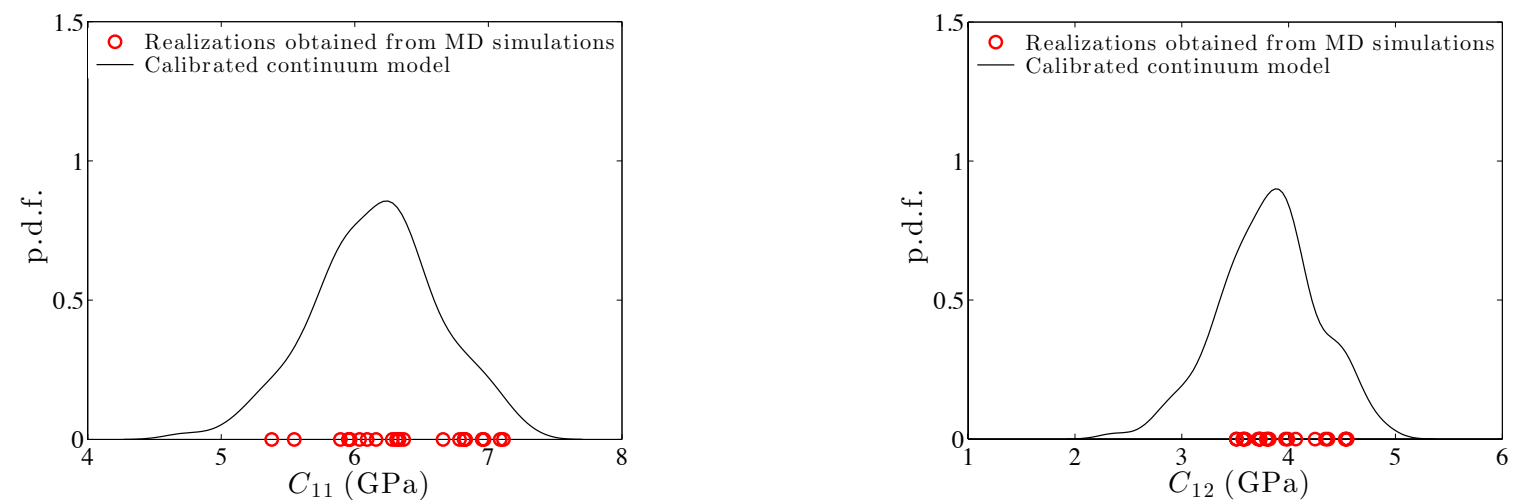

Figure 14: Plot of the estimated probability density function for the continuum model (solid line) and associated MD-based realizations (red circles). Left: component $C_{11}$. Right: component $C_{12}$.
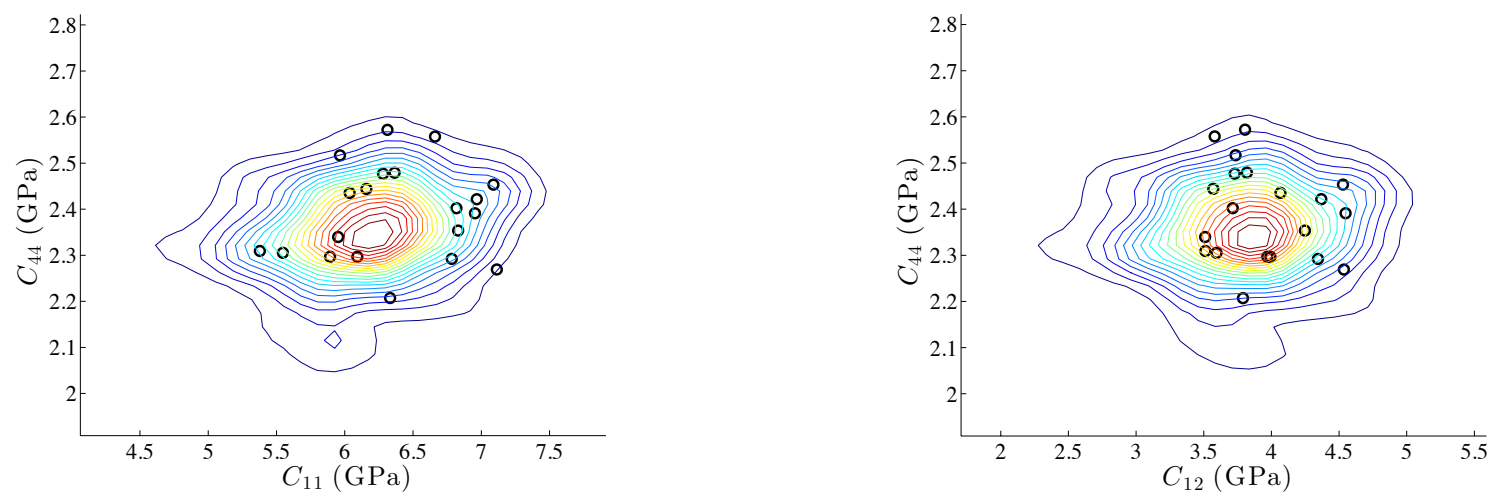

Figure 15: Plot of the estimated joint probability density function for the continuum model (contour plot) and associated MD-based realizations (black circles). Left: components $\left(C_{11}, C_{44}\right)$. Right: components $\left(C_{12}, C_{44}\right)$.

\subsection{Calibration with MD finite sampling noise}

In this section, the effect of MD finite sampling noise on calibration results is investigated. From a physical standpoint, it can be reasonably assumed that such a noise does not impact the correlation structure of the underlying elasticity random field in the interphase region, but may yield an overestimation of $\delta_{[\mathcal{N}]}$. Therefore, the sampling noise is taken into account below by modeling the spatially-constant but random properties of the bulk polymer and Silica inclusion by random matrices, and by considering a triclinic noise independent of $\boldsymbol{x}^{s}$ in the interphase elasticity random field written in spherical coordinates. Let $\left\{\left[\boldsymbol{C}_{W N}^{s}\left(\boldsymbol{x}^{s}\right)\right], \boldsymbol{x}^{s} \in \mathcal{D}_{\mathcal{I}}\right\}$ be the aforementioned random field in the interphase region. The same probabilistic model is used for all the random matrices and corresponds to the MaxEnt-based model proposed in [55]. For a given phase, the 
model depends on a prescribed mean value, as well as on a parameter controlling the level of statistical fluctuations. It is deduced from the atomistic simulations that the mean values for the polymer and Silica bulks are isotropic and defined by the properties listed at the end of section 4.1, whereas the mean for the random matrix modeling the noise in the interphase region is set to $\left[I_{6}\right]$ (so that triclinic fluctuations are introduced). It is further assumed that the random matrices depend on the same dispersion parameter, denoted by $\delta_{\text {noise }}$. Optimal value for parameters $\delta_{[\mathcal{N}]}$ and $\delta_{\text {noise }}$ are then defined as those maximizing the likelihood function

$$
\mathcal{L}_{3}\left(\delta_{[\mathcal{N}]}, \delta_{\text {noise }}\right)=\prod_{i=1}^{20} p_{\left[\widetilde{\boldsymbol{C}}_{\mathrm{CM}}\left(\delta_{[\mathcal{N}]}, \delta_{\text {noise }}\right)\right]}\left(\left[\widetilde{C}_{\mathrm{MD}}\left(\omega_{i}\right)\right]\right)
$$

over $\left[0, \sqrt{7 / 11}\left[\times\left[0, \sqrt{7 / 11}\left[\right.\right.\right.\right.$. In Eq. $(46),\left[\widetilde{\boldsymbol{C}}_{\mathrm{CM}}\left(\delta_{[\mathcal{N}]}, \delta_{\text {noise }}\right)\right]$ is the random variable modeling the apparent stiffness tensor obtained by homogenizing the random microstructure defined as follows:

- the random field $\left\{\left[\boldsymbol{C}_{W N}^{s}\left(\boldsymbol{x}^{s}\right)\right], \boldsymbol{x}^{s} \in \mathcal{D}_{\mathcal{I}}\right\}$ in the interphase region is written as

$$
\forall \boldsymbol{x}^{s} \in \mathcal{D}_{\mathcal{I}}, \quad\left[\boldsymbol{C}_{W N}^{s}\left(\boldsymbol{x}^{s}\right)\right]=\eta\left[I_{6}\right]+\left[\mathcal{M}^{s}\left(\boldsymbol{x}^{s}\right)\right]^{1 / 2}[\boldsymbol{Z}]\left[\mathcal{M}^{s}\left(\boldsymbol{x}^{s}\right)\right]^{1 / 2}
$$

where $\left\{\left[\mathcal{M}^{s}\left(\boldsymbol{x}^{s}\right)\right], \boldsymbol{x}^{s} \in \mathcal{D}_{\mathcal{I}}\right\}$ is defined by the vector $\boldsymbol{h}_{1}^{\text {opt }}$ and parameters $\left(L_{r}^{\mathrm{opt}}, L_{a}^{\mathrm{opt}}\right)$ calibrated in section 4.4 , as well as by $\delta_{[\mathcal{N}]}$ (to be updated); $[\boldsymbol{Z}]$ is the $\mathbb{M}_{6}^{+}(\mathbb{R})$-valued random matrix depending on $\delta_{\text {noise }}$ (to be calibrated) and such that $\mathbb{E}([\boldsymbol{Z}])=\left[I_{6}\right]$;

- the random properties in the bulk polymer and Silica particle defined by the mean values computed in section 4.1 and dispersion parameter $\delta_{\text {noise }}$.

The plot of the likelihood function $\left(\delta_{[\mathcal{N}]}, \delta_{\text {noise }}\right) \mapsto \mathcal{L}_{3}\left(\delta_{[\mathcal{N}]}, \delta_{\text {noise }}\right)$ is shown in Fig. 16. Optimal values are found to be $\delta_{[\mathcal{N}]}^{\text {opt }}=0.2$ and $\delta_{\text {noise }}^{\text {opt }}=0.1$, which shows that neglecting finite sampling noise for the MD system under consideration yields an overestimation of $\delta_{[\mathcal{N}]}$.

\section{Conclusion}

This study was devoted to the construction and identification, based on results arising from MD simulations, of a random field model for the elastic properties of the interphase region surrounding nanoscopic fillers. Atomistic simulations were performed on a prototypical nanocomposite and allowed some fundamental features of the random field to be inferred. A stochastic representation was then proposed, making use of a generalized formulation derived elsewhere. The stochastic field thus defined exhibits transverse isotropy in spherical coordinates (in accordance with the MD-predicted geometrical configuration of the polymer chain segments in the neighborhood of the nanoinclusion), and interestingly depends on a low-dimensional hyperparameter. An optimal 


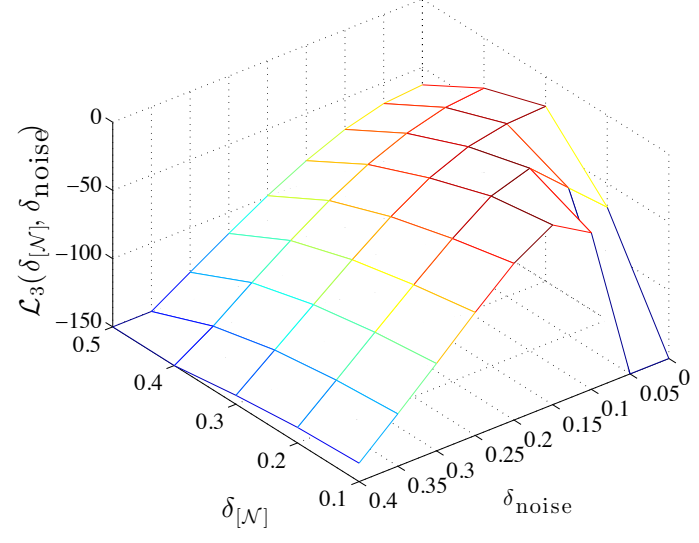

Figure 16: Plot of the likelihood function $\left(\delta_{[\mathcal{N}]}, \delta_{\text {noise }}\right) \mapsto \mathcal{L}_{3}\left(\delta_{[\mathcal{N}]}, \delta_{\text {noise }}\right)$. Former results (with no sampling noise) correspond to the curve $\delta_{[\mathcal{N}]} \mapsto \mathcal{L}_{3}\left(\delta_{[\mathcal{N}]}, 0\right)$.

value of this hyperparameter was then sought by solving a statistical inverse problem based on an upscaling procedure. It is shown that the elasticity random field exhibits nonnegligible fluctuations, and that the estimates of the spatial correlation lengths are actually consistent with characteristic lengths of the atomistic model. These results were finally refined by integrating fluctuations due to MD finite sampling noise, which allows for a better estimation of the parameter related to the level of fluctuations.

\section{Acknowledgments}

This work was supported by the French National Research Agency (ANR) under contract ANR12-JS09-0001-01.

\section{References}

[1] S.-Y. Fu, X.-Q. Feng, B. Lauke, Y.-W. Mai, Effects of particle size, particle/matrix interface adhesion and particle loading on mechanical properties of particulate-polymer composites, Composites: Part B 39 (2008) 933-961.

[2] J. Móczó, B. Pukánszky, Polymer micro and nanocomposites: Structure, interactions, properties, Journal of Industrial and Engineering Chemistry 14 (2008) 535-563.

[3] S. Harton, S. Kumar, H. Yang, T. Koga, K. Hicks, H. Lee, J. Mijovic, M. Liu, R. Vallery, D. Gidley, Immobilized polymer layers on spherical nanoparticles, Macromolecules 43 (2010) $3415-3421$. 
[4] A. Holt, P. Griffin, V. Bocharova, A. Agapov, A. Imel, M. Dadmun, J. Sangoro, A. Sokolov, Dynamics at the Polymer/Nanoparticle Interface in Poly(2-vinylpyridine)/Silica Nanocomposites, Macromolecules 47 (2014) 1837-1843.

[5] A. Papon, K. Saalwächter, K. Schäler, L. Guy, F. Lequeux, H. Montes, Low-Field NMR Investigations of Nanocomposites: Polymer Dynamics and Network Effects, Macromolecules 44 (2011) 913-922.

[6] D. Fragiadakis, L. Bokobza, P. Pissis, Dynamics near the filler surface in natural rubber-silica nanocomposites, Polymer 52 (2011) 3175-3182.

[7] Z. S. Petrović, Y. J. Cho, I. Javni, S. Magonov, N. Yerina, D. W. Schaefer, J. Ilavsky, A. Waddon, Effect of silica nanoparticles on morphology of segmented polyurethanes, Polymer 45 (12) (2004) 4285-4295.

[8] J. Berriot, F. Lequeux, L. Monnerie, H. Montes, D. Long, P. Sotta, Filler-elastomer interaction in model filled rubbers, a ${ }^{1} \mathrm{H}$ NMR study, Journal of Non-Crystalline Solids 307 (2002) 719-724.

[9] J. Berriot, F. Martin, H. Montes, L. Monnerie, P. Sotta, Reinforcement of model filled elastomers: characterization of the crosslinking density at the filler-elastomer interface by ${ }^{1} \mathrm{H}$ NMR measurements, Polymer 44 (5) (2003) 1437-1447.

[10] G. Leu, Y. Liu, D. Werstler, D. Cory, NMR Characterization of Elastomer-Carbon Black Interactions, Macromolecules 37 (2004) 6883-6891.

[11] D. Barbier, D. Brown, A. Grillet, S. Neyertz, Interface between End-Functionalized PEO Oligomers and a Silica Nanoparticle Studied by Molecular Dynamics Simulations, Macromolecules 37 (2004) 4695-4710.

[12] D. Brown, V. Marcadon, P. Mélé, N. D. Albérola, Effect of filler particle size on the properties of model nanocomposites, Macromolecules 41 (4) (2008) 1499-1511.

[13] D. Brown, P. Mélé, S. Marceau, N. D. Albérola, A molecular dynamics study of a model nanoparticle embedded in a polymer matrix, Macromolecules 36 (4) (2003) 1395-1406.

[14] F. Starr, T. Schrøder, S. Glotzer, Molecular Dynamics Simulation of a Polymer Melt with a Nanoscopic Particle, Macromolecules 35 (2002) 4481-4492.

[15] G. M. Odegard, T. C. Clancy, T. S. Gates, Modeling of the mechanical properties of nanoparticle/polymer composites, Polymer 46 (2) (2005) 553-562. 
[16] T. V. M. Ndoro, E. Voyiatzis, A. Ghanbari, D. N. Theodorou, M. C. Böhm, F. Müller-Plathe, Interface of grafted and ungrafted silica nanoparticles with a polystyrene matrix: Atomistic molecular dynamics simulations, Macromolecules 44 (7) (2011) 2316-2327.

[17] T. V. M. Ndoro, M. C. Böhm, F. Müller-Plathe, Interface and interphase dynamics of polystyrene chains near grafted and ungrafted silica nanoparticles, Macromolecules 45 (1) (2012) 171-179.

[18] A. Ghanbari, T. V. M. Ndoro, F. Leroy, M. Rahimi, M. C. Böhm, F. Müller-Plathe, Interphase structure in silica-polystyrene nanocomposites: A coarse-grained molecular dynamics study, Macromolecules 45 (1) (2012) 572-584.

[19] T. Chen, H. J. Qian, Y. L. Zhu, Z. Lu, Structure and Dynamics Properties at Interphase Region in the Composite of Polystyrene and Cross-Linked Polystyrene Soft Nanoparticle, Macromolecules 48 (2015) 2751-2760.

[20] M. Vacatello, Monte carlo simulations of polymer melts filled with solid nanoparticles, Macromolecules 34 (6) (2001) 1946-1952.

[21] M. Vacatello, Monte carlo simulations of the interface between polymer melts and solids. effects of chain stiffness, Macromolecular Theory and Simulations 10 (3) (2001) 187-195.

[22] M. Vacatello, Molecular arrangements in polymer-based nanocomposites, Macromolecular Theory and Simulations 11 (7) (2002) 757-765.

[23] M. Vacatello, Predicting the molecular arrangements in polymer-based nanocomposites, Macromolecular Theory and Simulations 12 (1) (2003) 86-91.

[24] G. G. Vogiatzis, E. Voyiatzis, D. N. Theodorou, Monte carlo simulations of a coarse grained model for an athermal all-polystyrene nanocomposite system, European Polymer Journal 47 (2011) 699-712.

[25] H. H.L. Duan, X. Yi, Z. Huang, J. Wang, A unified scheme for prediction of effective moduli of multiphase composites with interface effects. part i: Theoretical framework, Mechanics of Materials 39 (2007) 81-93.

[26] H. H.L. Duan, X. Yi, Z. Huang, J. Wang, A unified scheme for prediction of effective moduli of multiphase composites with interface effects. part ii: Application and scaling laws, Mechanics of Materials 39 (2007) 94-103.

[27] H. Le Quang, Q. C. He, Variational principles and bounds for elastic inhomogeneous materials with coherent imperfect interfaces, Mechanics of Materials 40 (10) (2008) 865-884. 
[28] H. Le Quang, Q. C. He, Size-dependent effective thermoelastic properties of nanocomposites with spherically anisotropic phases, Journal of the Mechanics and Physics of Solids 55 (9) (2007) 1899-1931.

[29] V. B. Shenoy, Atomistic calculations of elastic properties of metallic fcc crystal surfaces, Physical Review B 71 (2005) 094104.

[30] V. Marcadon, D. Brown, E. Herve, P. Mele, N. D. Alberola, A. Zaoui, Confrontation between molecular dynamics and micromechanical approaches to investigate particle size effects on the mechanical behaviour of polymer nanocomposites, Computational Materials Science 79 (2013) 495-505.

[31] B. Paliwal, M. Cherkaoui, Estimation of anisotropic elastic properties of nanocomposites using atomistic-continuum interphase model, International Journal of Solids and Structures 49 (18) (2012) 2424-2438.

[32] R. Peng, H. Zhou, H. Wang, L. Mishnaevsky Jr., Modeling of nano-reinforced polymer composites: Microstructure effect on Young's modulus, Computational Materials Science 60 (2012) $19-31$.

[33] S. Pfaller, M. Rahimi, G. Possart, P. Steinmann, F. Müller-Plathe, M. Böhm, An Arlequinbased method to couple molecular dynamics and finite element simulations of amorphous polymers and nanocomposites, Computer Methods in Applied Mechanics and Engineering 260 (2013) 109-129.

[34] N. Vu-Bac, T. Lahmer, Y. Zhang, X. Zhuang, T. Rabczuk, Stochastic predictions of interfacial characteristic of polymeric nanocomposites (PNCs), Composites: Part B 59 (2014) 80-95.

[35] N. Vu-Bac, T. Lahmer, H. Keitel, J. Zhao, X. Zhuang, T. Rabczuk, Stochastic predictions of bulk properties of amorphous polyethylene based on molecular dynamics simulations, Mechanics of Materials 68 (2014) 70-84.

[36] N. Vu-Bac, R. Rafiee, X. Zhuang, T. Lahmer, T. Rabczuk, Uncertainty quantification for multiscale modeling of polymer nanocomposites with correlated parameters, Composites: Part B 68 (2015) 446-464.

[37] N. Vu-Bac, M. Silani, T. Lahmer, X. Zhuang, T. Rabczuk, A unified framework for stochastic predictions of mechanical properties of polymeric nanocomposites, Computational Materials Science 96 (2015) 520-535. 
[38] F. Rizzi, H. N. Najm, B. J. Debusschere, K. Sargsyan, M. Salloum, H. Adalsteinsson, O. M. Knio, Uncertainty quantification in md simulations. part i: Forward propagation, Multiscale Modeling \& Simulation 10 (2012) 1428-1459.

[39] F. Rizzi, H. N. Najm, B. J. Debusschere, K. Sargsyan, M. Salloum, H. Adalsteinsson, O. M. Knio, Uncertainty quantification in md simulations. part ii: Bayesian inference of force-field parameters, Multiscale Modeling \& Simulation 10 (2012) 1460-1492.

[40] M. Salloum, K. Sargsyan, R. Jones, H. N. Debusschere, B. Najm, H. Adalsteinsson, A stochastic multiscale coupling scheme to account for sampling noise in atomistic-to-continuum simulations, Multiscale Modeling \& Simulation 10 (2012) 550-584.

[41] S. Chang, S. Yang, H. Shin, M. Cho, Multiscale homogenization model for thermoelastic behavior of epoxy-based composites with polydisperse $\mathrm{SiC}$ nanoparticles, Composite Structures 128 (2015) 342-353.

[42] H. Ghasemi, R. Rafiee, X. Zhuang, J. Muthu, T. Rabczuk, Uncertainties propagation in metamodel-based probabilistic optimization of CNT/polymer composite structure using stochastic multi-scale modeling, Computational Materials Science 85 (2014) 295-305.

[43] S. Plimpton, Fast parallel algorithms for short-range molecular-dynamics, Journal of Computational Physics 117 (1) (1995) 1-19.

[44] A. Stukowski, Visualization and analysis of atomistic simulation data with ovito-the open visualization tool, Modelling and Simulation in Materials Science and Engineering 18 (1) (2010) 015012 .

[45] S. Mayo, B. Olafson, W. Goddard, DREIDING: a generic force field for molecular simulations, The Journal of Physical Chemistry 94 (1990) 8897-8909.

[46] K. Binder, Monte Carlo and Molecular Dynamics Simulations in Polymer Science, Oxford University Press, 1995.

[47] M. P. Allen, D. J. Tildesley, Computer Simulation of Liquids, Clarendon Press Oxford, 1989.

[48] E. Voyiatzis, M. Rahimi, F. Müller-Plathe, M. Böhm, How thick is the polymer interphase in nanocomposites? probing it by local stress anisotropy and gas solubility, Macromolecules 47 (22) (2014) 7878-7889.

[49] W. Shinoda, M. Shiga, M. Mikami, Rapid estimation of elastic constants by molecular dynamics simulation under constant stress, Physical Review B 69 (2004) 134103. 
[50] J. Guilleminot, C. Soize, Stochastic model and generator for random fields with symmetry properties: Application to the mesoscopic modeling of elastic random media, Multiscale Modeling \& Simulation 11 (3) (2013) 840-870.

[51] L. J. Walpole, Fourth-rank tensors of the thirty-two crystal classes: Multiplication tables, Proc. R. Soc. Lond. A 391 (1984) 149-179.

[52] C. Soize, Non-gaussian positive-definite matrix-valued random fields for elliptic stochastic partial differential operators, Computer Methods in Applied Mechanics and Engineering 195 (1-3) (2006) 26-64.

[53] C. E. Shannon, A mathematical theory of communication, Bell System Technical Journal 27 (1948) 379-423/623-659.

[54] E. T. Jaynes, Information theory and statistical mechanics, Physical Review 106/108 (4/2) (1957) 620-630/171-190.

[55] C. Soize, A nonparametric model of random uncertainties for reduced matrix models in structural dynamics, Probabilistic Engineering Mechanics 15 (3) (2000) 277-294.

[56] B. Staber, J. Guilleminot, Approximate solutions of lagrange multipliers for informationtheoretic random field models, SIAM/ASA Journal on Uncertainty Quantification, accepted for publication on May 26, 2015.

[57] C. Soize, Construction of probability distributions in high dimension usign the maximum entropy principle: Applications to stochastic processes, random fields and random matrices, International Journal for Numerical Methods in Engineering 76 (2008) 1583-1611.

[58] L. Verlet, Computer "Experiments" on Classical Fluids. I. Thermodynamical Properties of Lennard-Jones Molecules, Physical Review 159 (1) (1967) 98-103.

[59] R. De Vogelaere, Methods of integration which preserve the contact transformation property of the Hamiltonian equations, Tech. Rep. 4, University of Notre Dame (1956).

[60] E. Hairer, C. Lubich, G. Wanner, Geometric Numerical Integration. Structure-Preserving Algorithms for Ordinary Differential Equations, Springer, Berlin, 2002.

[61] K. Burrage, I. Lenane, G. Lythe, Numerical methods for second-order stochastic differential equations, SIAM Journal on Scientific Computing 29 (1) (2007) 245-264.

[62] P. Kloeden, E. Platen, Numerical Solution of Stochastic Differentials Equations, Springer, Berlin, 1992. 
[63] G. Milstein, M. Tretyakov, Stochastic Numerics for Mathematical Physics, Springer, 2004.

[64] D. Talay, Simulation and Numerical Analysis of Stochastic Differential Systems: a Review, in: P. Kree, W. Wedig (Eds.), Probabilistic Methods in Applied Physics, Vol. 451 of Lecture Notes in Physics, Springer-Verlag, 1995, pp. 63-106.

[65] G. Papakonstantopoulos, M. Doxastakis, P. F. Nealey, J.-L. Barrat, J. de Pablo, Calculation of local mechanical properties of filled polymers, Physical Review E 75 (2007) 031803.

[66] E. Riccardi, M. C. Böhm, F. Müller-Plathe, Molecular dynamics method to locally resolve poisson's ratio: Mechanical description of the solid-soft-matter interphase, Physical Review E 86 (2012) 036704.

[67] C. Huet, Application of variational concepts to size effects in elastic heterogeneous bodies, Journal of the Mechanics and Physics of Solids 38 (1990) 813-841.

[68] M. Bornert, T. Bretheau, P. Gilormini, Homogenization in Mechanics of Materials, ISTE Publishing, 2008.

[69] M. Ostoja-Starzewski, Microstructural Randomness and Scaling in Mechanics of Materials, Chapman and Hall-CRC, 2008.

[70] M. Moakher, A. N. Norris, The closest elastic tensor of arbitrary symmetry to an elasticity tensor of lower symmetry, Journal of Elasticity 85 (2006) 215-263.

[71] M. Rahimi, I. Iriarte-Carretero, A. Ghanbari, M. C. Bohm, F. Muller-Plathe, Mechanical behavior and interphase structure in a silica-polystyrene nanocomposite under uniaxial deformation, Nanotechnology 23 (30). 